\title{
Covariants, derivation-invariant subsets, and first integrals
}

\author{
Frank Grosshans and Hanspeter Kraft
}

\begin{abstract}
Let $\mathbb{k}$ be an algebraically closed field of characteristic 0 , and let $V$ be a finite-dimensional vector space. Let $\operatorname{End}(V)$ be the semigroup of all polynomial endomorphisms of $V$. Let $\mathcal{E} \subseteq \operatorname{End}(V)$ be a linear subspace which is also a subsemigroup. Both $\operatorname{End}(V)$ and $\mathcal{E}$ are ind-varieties which act on $V$ in the obvious way.

In this paper, we study important aspects of such actions. We assign to $\mathcal{E}$ a linear subspace $\mathcal{D}_{\mathcal{E}}$ of the vector fields on $V$. A subvariety $X$ of $V$ is said to be $\mathcal{D}_{\mathcal{E}}$-invariant if $\xi(x) \in T_{x} X$ for all $\xi \in \mathcal{D}_{\mathcal{E}}$. We show that $X$ is $\mathcal{D}_{\mathcal{E}}$-invariant if and only if it is the union of $\mathcal{E}$-orbits. For such $X$, we define first integrals and show that they are the rational functions on a certain "quotient" of $X$ defined by the action of $\mathcal{E}$.

An important case occurs when $G$ is an algebraic subgroup of $\mathrm{GL}(V)$ and $\mathcal{E}$ consists of the G-equivariant polynomial endomorphisms. In this case, the associated $\mathcal{D}_{\mathcal{E}}$ is the space of $G$-invariant vector fields. A significant question here is whether there are non-constant $G$-invariant first integrals on $X$. As examples, we study the adjoint representation, Luna strata, the orbit closures of highest weight vectors, and representations of the additive group. We also look at finitedimensional irreducible representations of $\mathrm{SL}_{2}$ and their nullcones.
\end{abstract}

Keywords. Covariants; endomorphisms; invariant subsets; vector fields; first integrals; ind-varieties

2020 Mathematics Subject Classification. Primary: 14L30, 22E47; secondary: 13A50, 34C14

[Français]

Covariants, sous-ensembles invariants par dérivation et intégrales premières

Résumé. Soient $\mathbb{k}$ un corps algébriquement clos de caractéristique nulle et $V$ un espace vectoriel de dimension finie. Soit $\operatorname{End}(V)$ le semi-groupe des endomorphismes polynomiaux de $V$. Soit $\mathcal{E} \subseteq \operatorname{End}(V)$ un sous-espace linéaire qui est aussi un semi-groupe. Ainsi $\operatorname{End}(V)$ et $\mathcal{E}$ sont des ind-variétés qui agissent naturellement sur $V$.

Dans cet article, nous étudions des aspects importants de ces actions. Nous associons à $\mathcal{E}$ un sous-espace linéaire $\mathcal{D}_{\mathcal{E}}$ formés de champs de vecteurs sur $V$. Une sous-variété $X$ de $V$ est dite $\mathcal{D}_{\mathcal{E}}$-invariante si $\xi(x) \in T_{X} X$ pour tout $\xi \in \mathcal{D}_{\mathcal{E}}$. Nous montrons que $X$ est $\mathcal{D}_{\mathcal{E}}$-invariante si et seulement si elle est réunion de $\mathcal{E}$-orbites. Pour une telle sous-variété $X$, nous définissons des intégrales premières et montrons que ce sont des fonctions rationnelles sur un certain "quotient" de $X$ défini par l'action de $\mathcal{E}$.

Un cas particulier se présente lorsque $G$ est un sous-groupe algébrique de $\operatorname{GL}(V)$ et $\mathcal{E}$ est formé par les endomorphismes polynomiaux $G$-équivariants. Dans ce cas, $\mathcal{D}_{\mathcal{E}}$ est l'espace des champs de vecteurs $G$-invariants. Ici, une question naturelle porte sur l'existence d'intégrales premières $G$-invariantes non constantes sur $X$. Comme exemples, nous étudions la représentation adjointe, les strates de Luna, les adhérences d'orbites de vecteurs de plus haut poids et les représentations du groupe additif. Nous considérons aussi les représentations irréductibles de dimension finie de $\mathrm{SL}_{2}$ et leurs nilcônes.

Received by the Editors on December 16, 2019, and in final form on June 17, 2020.

Accepted on July 25, 2020.

Frank Grosshans

Department of Mathematics, West Chester University, West Chester, PA 19383, USA

e-mail: fgrosshans@wcupa.edu

Hanspeter Kraft

Departement Mathematik und Informatik, Universität Basel, Spiegelgasse 1, CH-4051 Basel, Switzerland

e-mail: Hanspeter.Kraft@unibas.ch

(C) by the author(s)

This work is licensed under http://creativecommons.org/licenses/by-sa/4.0/ 


\section{Contents}

1. Introduction and main results. . . . . . . . . . . . . . . . . . . . . . . . . 2

2. Basic material . . . . . . . . . . . . . . . . . . . . . . . . . . . . . . . 3

3. Endomorphisms . . . . . . . . . . . . . . . . . . . . . . . . . . . . . . 5

4. $\operatorname{Vec}_{G}$-symmetry . . . . . . . . . . . . . . . . . . . . . . . . . . . . . 8

5. First integrals . . . . . . . . . . . . . . . . . . . . . . . . . . . . . . . 12

6. Actions of $\mathrm{SL}_{2}$. . . . . . . . . . . . . . . . . . . . . . . . . . . . . . . 17

Appendix: Ind-varieties and ind-semigroups . . . . . . . . . . . . . . . . . . . . . 24

References. . . . . . . . . . . . . . . . . . . . . . . . 26

\section{Introduction and main results}

This paper is concerned with the relationship between three concepts: derivation-invariant subsets, endomorphisms of an affine variety $X$, and first integrals. We show that this relationship has features similar to those of algebraic group actions with first integrals playing the role of invariant functions. Let $\mathbb{k}$ be an algebraically closed field of characteristic 0 and let $X$ be an irreducible affine variety over $\mathrm{k}$. Let $\mathcal{D} \subseteq \operatorname{Vec}(X)$ be a set of algebraic vector fields on $X$. A closed subvariety $Y \subseteq X$ is called $\mathcal{D}$-invariant if $\xi(y) \in T_{y} Y$ for all $y \in Y$ and $\xi \in \mathcal{D}$, i.e., $\xi$ is tangent to $Y$ at every point of $Y$. We establish some basic properties of invariant subsets including the following: For any $x \in X$, there is a smallest $\mathcal{D}$-invariant closed subvariety, $M(x)$, which contains $x$ (Lemma 2.5). A first integral of $\mathcal{D}$ is a rational function $f \in \mathbb{k}(X)$ such that $\xi f=0$ for all $\xi \in \mathcal{D}$. We show that first integrals are precisely those functions which are constant on the closed subsets $M(x)$ (Lemma 5.2).

Our new idea is to consider the semigroup $\operatorname{End}(X)$ consisting of all endomorphisms of the variety $X$ and to use the important fact that $\operatorname{End}(X)$ is a so-called ind-variety. This allows us to define the (Zariski) tangent space $T_{\mathrm{id}} \operatorname{End}(X)$ and to associate to any $A \in T_{\mathrm{id}} \operatorname{End}(X)$ a vector field $\xi_{A}$ on $X$ in the usual way, see Section 3.1. For a closed subsemigroup $\mathcal{E} \subseteq \operatorname{End}(X)$ we denote by $\mathcal{D}_{\mathcal{E}} \subseteq \operatorname{Vec}(X)$ the set of associated vector fields. There is a natural action of $\operatorname{End}(X)$ on $X,(\varphi, x) \mapsto \varphi(x)$, and the $\mathcal{E}$-orbit of an element $x \in X$ is defined as $\mathcal{E}(x):=\{\varphi(x) \mid \varphi \in \mathcal{E}\}$. We first show that if a closed subvariety $Y \subseteq X$ is the union of $\mathcal{E}$-orbits, then $Y$ is $\mathcal{D}_{\mathcal{E}}$-invariant (Proposition 3.3).

Now suppose that $V$ is a finite-dimensional vector space and that $X \subseteq V$. Suppose also that $\mathcal{E} \subseteq \operatorname{End}(X)$ is a linear subspace which is also a subsemigroup. We show that for $x \in X, \mathcal{E}(x)=M(x)$ and that a subvariety $Y \subseteq X$ is $\mathcal{D}_{\mathcal{E}}$-invariant if and only if it is a union of $\mathcal{E}$-orbits (Theorem 3.8). This means that the $\mathcal{D}_{\mathcal{E}}$-invariant subvarieties are precisely those which are stable under the action of $\mathcal{E}$.

Furthermore, there is an open dense subset $X^{\prime} \subseteq X$ so that first integrals separate the various $\mathcal{E}(x) \cap X^{\prime}$ and thus can be regarded as the rational functions on a certain "quotient space" $X / / \mathcal{E}$ for the action of $\mathcal{E}$ on $X$ (Proposition 5.5). This construction includes an algebraic (and global) version of a classical theorem of Frobenius [War71, Theorem 1.60]. 
The most important example of the above occurs when $V$ is a finite-dimensional vector space, $G \subseteq \mathrm{GL}(V)$ an algebraic group,

$$
\operatorname{End}_{G}(V):=\{\varphi \in \operatorname{End}(V) \mid \varphi(g \cdot v)=g \cdot \varphi(v) \text { for all } g \in G \text { and } v \in V\},
$$

the semigroup of covariants, $X \subseteq V$ an $\operatorname{End}_{G}(V)$-stable closed subvariety and $\mathcal{E}:=\left.\operatorname{End}_{G}(V)\right|_{X}$. When $X$ is $G$-stable, an important question is whether or not there are non-constant $G$-invariant first integrals on $X$. Examples show that such can occur. However, in those cases where they do not, the field of first integrals is the field of rational functions on a homogeneous space (Lemma 5.8). Furthermore, the $\mathcal{E}$-G-orbit of a generic point is open and dense in $X$.

When $G$ is reductive and the orbit $G x$ is closed, Panyushev has shown that $\mathcal{E}(x)=X^{G_{x}}$ (see Proposition 4.10). Thus, first integrals separate open subsets of the various $X^{G_{x}}$. Furthermore, when the generic $G$-orbit in $X$ is closed, we show that there are no $G$-invariant first integrals (Theorem 5.16).

Finally, in Section 6, we study the case where $G=\mathrm{SL}_{2}$ and either $X=V_{d}$, the binary forms of degree $d$, or $X$ is the nullcone $\mathcal{N}_{d}$ of $V_{d}$. In the latter case, there are no closed orbits other than $\{0\}$ and the main problem in finding the $\mathcal{E}(v)$ is the construction of covariants.

Algebraically, derivation-invariant ideals have long been of interest [Sei67]. In the context of ordinary differential equations, it is well known that a Zariski-closed set $X \subseteq V$ is $\mathcal{D}_{\mathcal{E}}$-invariant if and only if it is the union of trajectories of solutions to $\frac{d x}{d t}=\xi(x), \xi \in \mathcal{E}$ (see Lemma 2.1 below). The study of the subsets $\mathcal{E}(v)$ began with the paper [LS99] by Lehrer-Springer which was subsequently extended by Panyushev [Pan02]. These papers, however, did not draw the connection to derivation-invariant subsets. For vector spaces, that connection appears in [GSW12, Theorem 3.6]. The difficult problem of constructing the module of covariants on $X$ was first considered in the classical invariant theory of the nineteenth century [Ell64] and continues to be of active research interest. It is worth noting that when $G$ is reductive and $X \subseteq V$ is an $\operatorname{End}_{G}(V)$ - and $G$-stable variety, then $\max \{\operatorname{dim} \mathcal{E}(v) \mid v \in X\}$, which is calculated in many of our examples, is (easily) shown to be the rank of the module of covariants from $X$ to $X$.

Acknowledgments. The first author thanks Sebastian Walcher for his advice on an earlier version of this paper.

\section{Basic material}

\subsection{Vector fields and $\mathcal{D}$-invariant subsets}

Our base field $\mathbb{k}$ is algebraically closed of characteristic zero. We start with a lemma which translates the concept of invariant subsets with respect to an ordinary differential equation into the algebraic setting. For an affine variety $X$ an algebraic vector field $\xi=(\xi(x))_{x \in X}$ is a collection of tangent vectors $\xi(x) \in T_{x} X$ such that, for every regular function $f \in \mathcal{O}(X)$, the function $\xi f: x \mapsto \xi(x) f$ is again regular. It is easy to see that this is the same as a derivation of the coordinate $\operatorname{ring} \mathcal{O}(X)$. Note that $\xi f$ is also defined for rational functions $f$.

In addition, one can define the the tangent bundle $T X$ of $X$ which is a variety together with a projection $p: T X \rightarrow X$ such that the fibers $p^{-1}(x)$ are the Zariski tangent spaces $T_{x} X$. Then the sections are the algebraic vector fields (see e.g. [Kra16, Appendix A.4.5]). It is clear that the algebraic vector fields form a $\mathcal{O}(X)$-module which will be denoted by $\operatorname{Vec}(X)$ and which can be identified with the $\mathcal{O}(X)$-module $\operatorname{Der}(\mathcal{O}(X))$ of derivations of $\mathcal{O}(X)$. The next lemma seems to be well known; a somewhat different proof may be found in [SSW15, Lemma A.1].

Lemma 2.1. Let $X$ be a smooth complex variety, and let $\xi \in \operatorname{Vec}(X)$ be an algebraic vector field. Then a Zariskiclosed subvariety $Y \subseteq X$ is invariant with respect to the flow defined by the differential equation $\dot{x}=\xi(x)$ if and only if $\xi(y) \in T_{y} Y$ for all $y \in Y$. 
Proof. Let $\Phi: X \times \mathbb{R} \rightarrow X$ be the local flow of $\xi$, defined in an open neighborhood of $X \times\{0\}$. By definition,

$$
\left.\frac{\partial}{\partial t} \Phi(x, t)\right|_{t=0}=\xi(x) \text { for all } x \in X \text {. }
$$

This implies that if $Y$ is invariant under $\Phi$, then $\xi(y) \in T_{y} Y$ for all $y \in Y$. On the other hand, assume that $\xi(y) \in T_{y} Y$ for all $y \in Y$, and denote by $Y^{\prime} \subseteq Y$ the open dense set of smooth points of $Y$. Then $\left.\xi\right|_{Y^{\prime}}$ defines a local flow $\Phi_{Y^{\prime}}: Y^{\prime} \times \mathbb{R} \rightarrow Y^{\prime}$ such that $\left.\frac{\partial}{\partial t} \Phi_{Y^{\prime}}\left(y^{\prime}, t\right)\right|_{t=0}=\xi\left(y^{\prime}\right)$ for all $y \in Y^{\prime}$. By the uniqueness of the local flow, we have $\Phi_{Y^{\prime}}=\left.\Phi\right|_{Y^{\prime} \times \mathbb{R}}$, and so $Y^{\prime}$ is invariant under $\Phi$. Since $Y=\overline{Y^{\prime}}$ we see that $Y$ is also invariant under $\Phi$.

This lemma allows to define the invariance of subvarieties with respect to a set of vector fields for an arbitrary $\mathbb{k}$-variety $X$.

Definition 2.2. Let $\mathcal{D} \subseteq \operatorname{Vec}(X)$ be a set of vector fields.

(1) A closed subvariety $Y \subseteq X$ is called $\mathcal{D}$-invariant if $\xi(y) \in T_{y} Y$ for all $y \in Y$ and all $\xi \in \mathcal{D}$. We also say that the vector fields $\xi \in \mathcal{D}$ are parallel to $Y$.

(2) A subspace $W \subseteq \mathcal{O}(X)$ is called $\mathcal{D}$-invariant if $\xi(W) \subseteq W$ for all $\xi \in \mathcal{D}$.

Remark 2.3. We will constantly use the following easy fact. If $\xi$ is a vector field parallel to $Y$ and $f$ a rational function on $X$ defined in a neighborhood $U$ of $y \in Y$, then $\xi(y) f=\xi(y)\left(\left.f\right|_{U \cap Y}\right)$. In particular, if $f$ is regular on $X$, then $\left.(\xi f)\right|_{Y}=\left.\xi\right|_{Y}\left(\left.f\right|_{Y}\right)$.

\section{2. $\mathcal{D}$-invariant ideals}

Let $\mathcal{D} \subseteq \operatorname{Vec}(X)$ be a set of vector fields.

Lemma 2.4. If $I(Y) \subseteq \mathcal{O}(X)$ denotes the vanishing ideal of $Y$, then $Y$ is $\mathcal{D}$-invariant if and only if $I(Y)$ is $\mathcal{D}$-invariant.

Proof. If $f \in I(Y)$, then, for $y \in Y,(\xi f)(y)=\xi(y) f=\left.\xi(y) f\right|_{Y}=0$, hence $\xi f \in I(Y)$. Conversely, if $\xi(I(Y)) \subseteq I(Y)$, then $\xi$ induces a derivation of $\mathcal{O}(X) / I(Y)=\mathcal{O}(Y)$, and the claim follows.

For a closed subvariety $Y \subseteq X$ we can define the Lie subalgebra of the vector fields on $X$ parallel to $Y$ :

$$
\operatorname{Vec}_{Y}(X):=\left\{\xi \in \operatorname{Vec}(X) \mid \xi(y) \in T_{y} Y \text { for all } y \in Y\right\} \subseteq \operatorname{Vec}(X) .
$$

We have a homomorphism of Lie algebras

$$
\rho: \operatorname{Vec}_{Y}(X) \rightarrow \operatorname{Vec}(Y),\left.\quad \xi \mapsto \xi\right|_{Y},
$$

whose kernel consists of the vector fields on $X$ vanishing on $Y$. The homomorphism $\rho$ is surjective when $X$ is a vector space. With this notation we see that $Y$ is $\mathcal{D}$-invariant if and only if $\mathcal{D} \subseteq \operatorname{Vec}_{Y}(X)$. Part (3) of the next lemma can be found in [Sei67, Theorem 1].

\section{Lemma 2.5.}

(1) Sums and intersections of $\mathcal{D}$-invariant ideals are $\mathcal{D}$-invariant.

(2) If $I \subseteq \mathcal{O}(X)$ is a $\mathcal{D}$-invariant ideal, then so is $\sqrt{I}$.

(3) If $Y_{i} \subseteq X, i \in I$, are $\mathcal{D}$-invariant closed subvarieties, then so is $\bigcap_{i \in I} Y_{i}$.

(4) For any $x \in X$ there is a uniquely defined minimal D-invariant closed subvariety $M(x) \subseteq X$ containing $x$.

(5) If the closed subvariety $Y \subseteq X$ is $\mathcal{D}$-invariant, then every irreducible component of $Y$ is $\mathcal{D}$-invariant. 
Proof. (1) is clear, (3) follows from (1) and (2), and (4) follows from (3).

(2) It suffices to show that if $f^{n}=0$, then $(\xi f)^{m}=0$ for some $m>0$. Let $e_{0} \geq 0$ be the minimal $e$ such that there exists a $q \geq 0$ with $f^{e} \cdot(\xi f)^{q}=0$. If $e_{0}=0$, we are done. So assume that $e_{0}>0$. Then

$$
0=\xi\left(f^{e_{0}} \cdot(\xi f)^{q}\right) \cdot \xi f=e_{0} f^{e_{0}-1} \cdot(\xi f)^{q+1}+q f^{e_{0}} \cdot(\xi f)^{q} \cdot \xi^{2} f=e_{0} f^{e_{0}-1} \cdot(\xi f)^{q+1},
$$

contradicting the minimality of $e_{0}$.

(5) It suffices to consider the case where $Y=X$, hence $(0)=\mathfrak{p}_{1} \cap \ldots \cap \mathfrak{p}_{k}$ where the $\mathfrak{p}_{i}$ are the minimal primes of $\mathcal{O}(X)$. For every $i$ choose an element $p_{i} \in \bigcap_{j \neq i} \mathfrak{p}_{j} \backslash \mathfrak{p}_{i}$. Then $\mathfrak{p}_{i}=\left\{p \in \mathcal{O}(X) \mid p_{i} p=0\right\}$, and the same holds for every power of $p_{i}$. For every $p \in \mathfrak{p}_{i}$ we find

$$
0=p_{i} \xi\left(p_{i} p\right)=p_{i}\left(p_{i} \xi p+p \xi p_{i}\right)=p_{i}^{2} \xi p,
$$

hence $\xi p \in \mathfrak{p}_{i}$.

Definition 2.6. The closed subvarieties $M(x) \subseteq X$ from Lemma 2.5(4) are called minimal D-invariant subvarieties. By Lemma 2.5(5) they are irreducible.

\subsection{Linear spaces of vector fields}

In the following, we will mainly deal with the case where $\mathcal{D} \subseteq \operatorname{Vec}(X)$ is a linear subspace. In this case, we set

$$
\mathcal{D}(x):=\varepsilon_{x}(\mathcal{D}):=\{\xi(x) \mid \xi \in \mathcal{D}\} \subseteq T_{x} X
$$

where $\varepsilon_{x}: \operatorname{Vec}(X) \rightarrow T_{x} X$ is the (linear) evaluation map $\xi \mapsto \xi(x)$. Note that a closed subvariety $Y \subseteq X$ is $\mathcal{D}$-invariant if and only if $\mathcal{D}(y) \subseteq T_{y} Y$ for all $y \in Y$.

The following lemma is clear.

Lemma 2.7. For a linear subspace $\mathcal{D} \subseteq \operatorname{Vec}(X)$ the function $x \mapsto \operatorname{dim} \mathcal{D}(x)$ is lower semicontinuous, i.e., for every $x \in X$ the set

$$
U_{x}:=\{u \in X \mid \operatorname{dim} \mathcal{D}(u) \geq \operatorname{dim} \mathcal{D}(x)\}
$$

is a (Zariski-) open neighborhood of $x$.

Setting $d_{\mathcal{D}}(X):=\max _{x \in X} \operatorname{dim} \mathcal{D}(x)$ the lemma implies that

$$
X^{\prime}:=\left\{x \in X \mid \operatorname{dim} \mathcal{D}(x)=d_{\mathcal{D}}(X)\right\}
$$

is open (and non-empty) in $X$.

\section{Endomorphisms}

\subsection{The semigroup of endomorphisms}

We now study the semigroup $\operatorname{End}(X)$ of endomorphisms of $X$. An important fact is that $\operatorname{End}(X)$ is an ind-variety (see Appendix) which allows to define the (Zariski) tangent space $T_{\mathrm{id}} \operatorname{End}(X)$. We have a canonical inclusion

$$
\Xi: T_{\mathrm{id}} \operatorname{End}(X) \hookrightarrow \operatorname{Vec}(X), A \mapsto \xi_{A},
$$

where the vector field $\xi_{A}$ is defined in the following way (see Appendix, Proposition A.5). For any $x \in X$ consider the "orbit map" $\mu_{x}: \operatorname{End}(X) \rightarrow X, \varphi \mapsto \varphi(x)$, and its differential

$$
\left(d \mu_{x}\right)_{\mathrm{id}}: T_{\mathrm{id}} \operatorname{End}(X) \rightarrow T_{x} X .
$$

Then define $\xi_{A}(x):=\left(d \mu_{x}\right)_{\text {id }}(A)$. 
The canonical "evaluation map" $(\varphi, x) \mapsto \varphi(x)$ defines a morphism of ind-varieties

$$
\Phi: \operatorname{End}(X) \times X \rightarrow X, \quad(\varphi, x) \mapsto \varphi(x)
$$

with the usual properties:

$$
\Phi(\mathrm{id}, x)=x \text { and } \Phi(\varphi \circ \psi, x)=\Phi(\varphi, \Phi(\psi, x))
$$

for all $x \in X$ and $\varphi, \psi \in \operatorname{End}(X)$. We will call this an action of the semigroup $\operatorname{End}(X)$ on $X$, although there are some major differences to group actions as we will see below.

For the differential of $\Phi$ we find

$$
d \Phi_{\left(\mathrm{id}, x_{0}\right)}: T_{\mathrm{id}} \operatorname{End}(X) \oplus T_{x_{0}} X \rightarrow T_{x_{0}} X, \quad(A, \delta) \mapsto \xi_{A}\left(x_{0}\right)+\delta .
$$

Definition 3.1. If $\mathcal{E} \subseteq \operatorname{End}(X)$ is a closed subsemigroup we say that a subset $Y \subseteq X$ is stable under $\mathcal{E}$ (shortly $\mathcal{E}$-stable), if $\varphi(y) \in Y$ for all $y \in Y$ and all $\varphi \in \mathcal{E}$.

Remark 3.2. The stability under $\mathcal{E}$ can be expressed differently by using the subsets

$$
\mathcal{E}(y):=\{\varphi(y) \mid \varphi \in \mathcal{E}\}
$$

which will be called orbits of $y$ under $\mathcal{E}$. Namely, $X$ is stable under $\mathcal{E}$ if and only if $X$ contains with every $y$ the orbit $\mathcal{E}(y)$. However, one has to be very careful in using this analogy with group actions since orbits under $\mathcal{E}$ are not necessarily disjoint and they do not define a partition of $X$.

The closed subsemigroup $\mathcal{E} \subseteq \operatorname{End}(X)$ defines a linear subspace $\mathcal{D}_{\mathcal{E}} \subseteq \operatorname{Vec}(X)$ as the image of the tangent space $T_{\text {id }} \mathcal{E}$ under $\Xi$ :

$$
\mathcal{D}_{\mathcal{E}}:=\left\{\xi_{A} \mid A \in T_{\text {id }} \mathcal{E}\right\} \subseteq \operatorname{Vec}(X) .
$$

The main point of this section is to relate the invariance under $\mathcal{D}_{\mathcal{E}}$ with the stability under the semigroup $\mathcal{E}$. A first and easy result is the following.

Proposition 3.3. Let $\mathcal{E} \subseteq \operatorname{End}(X)$ be a closed subsemigroup. If $Y \subseteq X$ is a closed $\mathcal{E}$-stable subvariety, then $Y$ is $\mathcal{D}_{\mathcal{E}}$-invariant.

Proof. Since $Y$ is $\mathcal{E}$-invariant we have a morphism $\Phi: \mathcal{E} \times Y \rightarrow Y$ whose differential

$$
d \Phi_{(\mathrm{id}, y)}: T_{\mathrm{id}} \mathcal{E} \oplus T_{y} Y \rightarrow T_{y} Y
$$

sends $(A, 0)$ to $\xi_{A}(y)$, by formula $(*)$ above. Thus $\xi(y) \in T_{y} Y$ for all $\xi \in \mathcal{D}_{\mathcal{E}}$ which means that $Y$ is $\mathcal{D}_{\mathcal{E}}$-invariant.

We will see below that under stronger assumptions on $\mathcal{E}$ the reverse implication also holds, i.e., a closed subset $Y \subseteq X$ is $\mathcal{E}$-stable if and only if it is $\mathcal{D}_{\mathcal{E}}$-invariant.

Remark 3.4. We do not know what the structure of the subsets $\mathcal{E}(x) \subseteq X$ is. If $\mathcal{E}$ is curve-connected (i.e. any two points of $\mathcal{E}$ can be connected by an irreducible curve, see Definition A.3(5)), then one can show that $\mathcal{E}(x)$ contains a set $U$ which is open and dense in $\overline{\mathcal{E}(x)}$. But it is not clear whether $\mathcal{E}(x)$ is constructible.

\subsection{The case of a vector space}

In case of a vector space $X=V$ the situation is much simpler, because we can identify every tangent space $T_{v} V$ with $V$. In particular, vector fields $\xi \in \operatorname{Vec}(V)$ correspond to morphisms $\xi: V \rightarrow V$. Choosing a basis of $V$ we have

$$
\xi=\sum_{i=1}^{n} p_{i} \frac{\partial}{\partial x_{i}} \text { where } p_{i}:=\xi x_{i}
$$

In this situation, the semigroup $\operatorname{End}(V)=\mathcal{O}(V) \otimes V$ is a vector space, hence $T_{\text {id }} \operatorname{End}(V)=\operatorname{End}(V)$ in a canonical way, and

$$
\Xi: \operatorname{End}(V)=T_{\mathrm{id}} \operatorname{End}(V) \stackrel{\sim}{\rightarrow} \operatorname{Vec}(V)
$$


is the obvious isomorphism given as follows. In terms of coordinates an endomorphism $\varphi$ has the form $\varphi=\left(p_{1}, \ldots, p_{n}\right): \mathbb{k}^{n} \rightarrow \mathbb{k}^{n}$ where $p_{i}=\varphi^{*}\left(x_{i}\right)$, and the corresponding vector field $\xi:=\Xi(\varphi)$ is given by $\xi=\sum_{i=1}^{n} p_{i} \frac{\partial}{\partial x_{i}}$.

The same formula holds for a semigroup $\mathcal{E} \subseteq \operatorname{End}(V)$ which is a linear subspace. However, for a general closed semigroup $\mathcal{E} \subseteq \operatorname{End}(V)$, we cannot identify $\mathcal{E}$ with $T_{\mathrm{id}} \mathcal{E}$, and so the formula above does not make sense. For example, if $\varphi \in \operatorname{End}(V)$ is any endomorphism, then the semigroup $\mathcal{E}:=\left\{\mathrm{id}, \varphi, \varphi^{2}, \ldots\right\}$ is discrete, hence $T_{\mathrm{id}} \mathcal{E}$ is trivial, and so $\mathcal{D}_{\mathcal{E}}$ is also trivial.

The following result is crucial. We will identify $T_{v} V$ with $V$ and thus consider the subspace $\mathcal{D}(v) \in T_{v} V$ as a subspace of $V$.

Lemma 3.5. Let $\mathcal{E} \subseteq \operatorname{End}(V)$ be a linear subspace which is a semigroup. Then

$$
\mathcal{E}(v)=M(v)=\mathcal{D}_{\mathcal{E}}(v) \text { for all } v \in V .
$$

In particular, a subset $Y \subseteq V$ is $\mathcal{D}_{\mathcal{E}}$-invariant if and only if it is $\mathcal{E}$-stable. Moreover, we have $\mathcal{E}(w)=\mathcal{E}(v)$ for all $w$ in an open neighborhood of $v$ in $\mathcal{E}(v)$.

Proof. (a) We have seen in Proposition 3.3 that $\mathcal{E}(v)$ is $\mathcal{D}_{\mathcal{E}}$-invariant, because it is stable under $\mathcal{E}$. Hence, $\mathcal{D}_{\mathcal{E}}(w) \subseteq T_{w} \mathcal{E}(v)$ for all $w \in \mathcal{E}(v)$.

(b) The evaluation map $\mu_{v}: \mathcal{E} \rightarrow V$ is linear with image $\mathcal{E}(v)$, hence $\mathcal{E}(v) \subseteq V$ is a linear subspace and $\mathcal{D}_{\mathcal{E}}(v)=T_{v} \mathcal{E}(v)=\mathcal{E}(v)$.

(c) By Lemma 2.7 there is an open neighborhood $U_{v}$ of $v$ in $\mathcal{E}(v)$ such that $\operatorname{dim} \mathcal{D}_{\mathcal{E}}(w) \geq \operatorname{dim} \mathcal{D}_{\mathcal{E}}(v)$ for all $w \in U_{v}$. Hence, $\mathcal{E}(v)=\mathcal{D}_{\mathcal{E}}(v)=\mathcal{D}_{\mathcal{E}}(w)=\mathcal{E}(w)$ for $w \in U_{v}$, by (a).

(d) It remains to prove the minimality, i.e. that $\mathcal{E}(v)=M(v)$. Let $Y \subseteq \mathcal{E}(v)$ be closed and $\mathcal{D}_{\mathcal{E}}$-invariant with $v \in Y$. Then, for every $w \in U_{v} \cap Y$, we have $\mathcal{E}(v)=\mathcal{D}_{\mathcal{E}}(w) \subseteq T_{w} Y \subseteq \mathcal{E}(v)$. Hence, $\operatorname{dim} Y \geq \operatorname{dim} \mathcal{E}(v)$, and so $Y=\mathcal{E}(v)$.

Remark 3.6. If $\mathcal{E} \subseteq \operatorname{End}(V)$ is as in the lemma above, then it contains the scalar multiplication $\mathbb{k} \cdot \mathrm{id}$, and so $\mathcal{E}(v) \supset \mathbb{k} v$ for all $v \in V$. Therefore, every $\mathcal{D}_{\mathcal{E}}$-invariant closed subvariety $X$ is a closed cone, i.e., contains with every point $x \neq 0$ the line $\mathrm{k} \cdot x$, and every $\mathcal{D}_{\mathcal{E}}$-invariant ideal is homogeneous.

\subsection{Linear semigroups}

One would like to extend the lemma above to a statement of the form that a subvariety $Y \subseteq X$ is stable under a closed semigroup $\mathcal{E} \subseteq \operatorname{End}(X)$ if and only if it is $\mathcal{D}_{\mathcal{E}}$-invariant where $\mathcal{D}_{\mathcal{E}}$ is the image of $T_{\text {id }} \mathcal{E}$ in $\operatorname{Vec}(X)$. We do not know if such a result holds in general, but we can prove it for so-called linear semigroups $\mathcal{E} \subseteq \operatorname{End}(X)$ which is sufficient for the applications we have in mind.

If $X \subseteq V$ is a closed subvariety, then $\operatorname{End}(X) \subseteq \operatorname{Mor}(X, V)$. Thus we can form linear combinations of endomorphisms of $X$, but in general the resulting morphism does not have its image in $X$.

Definition 3.7. A subsemigroup $\mathcal{E} \subseteq \operatorname{End}(X)$ is called linear if there is a closed embedding $X \hookrightarrow V$ into a vector space $V$ such that the image of $\mathcal{E}$ in $\operatorname{Mor}(X, V)$ is a linear subspace.

Theorem 3.8. Let $X$ be an affine variety, and let $\mathcal{E} \subseteq \operatorname{End}(X)$ be a linear semigroup.

(1) For any $x \in X$ we have $\mathcal{E}(x)=M(x)$.

(2) The subsets $\mathcal{E}(x) \subseteq X$ are closed and isomorphic to vector spaces.

(3) $T_{x} M(x)=T_{x} \mathcal{E}(x)=\mathcal{D}_{\mathcal{E}}(x)$ for all $x \in X$.

In particular, a closed subvariety $Y \subseteq X$ is $\mathcal{D}_{\mathcal{E}}$-invariant if and only if it is $\mathcal{E}$-stable, i.e. it is a union of $\mathcal{E}$-orbits. 
Proof. Choose a closed embedding $X \subseteq V$ such that $\mathcal{E} \subseteq \operatorname{Mor}(X, V)$ is a linear subspace. Since the map $\operatorname{End}(V) \rightarrow \operatorname{Mor}(X, V)$ is linear and surjective there is a linear subspace $\tilde{\mathcal{E}} \subseteq \operatorname{End}(V)$ whose image in $\operatorname{Mor}(X, V)$ is $\mathcal{E}$. In particular, $X$ is stable under $\tilde{\mathcal{E}}$ and so $\mathcal{D}_{\tilde{\mathcal{E}}} \subseteq \operatorname{Vec}_{X}(V)$. The linearity of the map $\operatorname{End}(V) \rightarrow \operatorname{Mor}(X, V)$ implies that the image of $\mathcal{D}_{\tilde{\mathcal{E}}}$ under $\operatorname{Vec}_{X}(V) \rightarrow \operatorname{Vec}(X)$ is $\mathcal{D}_{\mathcal{E}}$, i.e. $\mathcal{D}_{\tilde{\mathcal{E}}}(x)=\mathcal{D}_{\mathcal{E}}(x)$ for all $x \in X$.

Now we apply Lemma 3.5 to $\tilde{\mathcal{E}}$ and find that $\mathcal{E}(x)=\tilde{\mathcal{E}}(x)=M(x)$, hence (1) and (2). Moreover, we have $T_{x} \mathcal{E}(x)=T_{x} \tilde{\mathcal{E}}(x)=\tilde{\mathcal{E}}(x)=\mathcal{D}_{\tilde{\mathcal{E}}}(x)=\mathcal{D}_{\mathcal{E}}(x)$, hence (3).

Finally, for a closed subvariety $Y \subseteq X \subseteq V$ the $\mathcal{D}_{\mathcal{E}}$-invariance is the same as the $\mathcal{D}_{\tilde{\mathcal{E}}}$-invariance, and $Y$ is stable under $\tilde{\mathcal{E}}$ if and only if it is stable under $\mathcal{E}$. Hence the last claim follows also from the lemma.

If $\mathcal{E} \subseteq \operatorname{End}(X)$ is a linear semigroup we define $d_{\mathcal{E}}(X):=\max _{x \in X} \operatorname{dim} \mathcal{E}(x)$. By our theorem above we have $d_{\mathcal{E}}(X)=d_{\mathcal{D}_{\mathcal{E}}}(X)$.

Corollary 3.9. Let $X$ be an irreducible variety and $\mathcal{E} \subseteq \operatorname{End}(X)$ a linear semigroup. The subset defined by $X^{\prime}:=\left\{x \in X \mid \operatorname{dim} \mathcal{E}(x)=d_{\mathcal{E}}(X)\right\}$ is then open and dense in $X$, and the subsets $\mathcal{E}(x) \cap X^{\prime}$ for $x \in X^{\prime}$ form a partition of $X^{\prime}$.

Proof. The first part is Lemma 2.7. If $y \in \mathcal{E}(x)$, then $\mathcal{E}(y) \subseteq \mathcal{E}(x)$. Since, by the theorem above, the $\mathcal{E}(x)$ are vector spaces and $\operatorname{dim} \mathcal{E}(x)=\operatorname{dim} \mathcal{D}_{\mathcal{E}}(x)$, we have $\mathcal{E}(y)=\mathcal{E}(x)$ in case $y \in X^{\prime}$. This proves the second claim.

Remark 3.10. If an algebraic group $G$ acts on a variety $X$, then every element $A \in \operatorname{Lie} G$ defines a vector field $\xi_{A}$. It is known that for a connected group $G$ a closed subvariety $Y \subseteq X$ is $G$-stable if and only if $Y$ is $\xi_{A}$-invariant for all $A \in \operatorname{Lie} G$. A proof can be found in [Kra16, III.4.4, Corollary 4.4.7], and the generalization to actions of connected ind-groups on affine varieties is given in [FK18, Proposition 7.2.6]. Our main theorem above shows that a similar statement holds for linear semigroups.

Remark 3.11. Let $G$ be a reductive group acting on an affine variety $X$. Denote by $\pi: X \rightarrow X / / G$ the algebraic quotient, i.e. the morphism defined by the inclusion $\mathcal{O}(X)^{G} \hookrightarrow \mathcal{O}(X)$. It is then clear that every $G$-invariant vector field on $X$ induces a vector field on the quotient $X / / G$. Schwarz shows in [Sch13] that if the induced map $\operatorname{Vec}(X)^{G} \rightarrow \operatorname{Vec}(X / / G)$ is surjective, then the Luna strata of the quotient $X / / G$ are intrinsic, i.e. they are permuted by all automorphisms of $X / / G$. We will prove a similar statement in Section 4.6 with the methods developed in this paper.

\section{4. $\operatorname{Vec}_{G}$-symmetry}

\subsection{G-equivariant endomorphisms}

Now consider an action of an algebraic group $G$ on the affine variety $X$. Then the induced actions of $G$ on the coordinate ring $\mathcal{O}(X)$ and on the vector fields $\operatorname{Vec}(X)$ are locally finite and rational, and the $G$-invariant vector fields $\operatorname{Vec}_{G}(X)$ form an $\mathcal{O}(V)^{G}$-module. Note that the (linear) action of $G$ on $\operatorname{Vec}(X)$ is given by $g \xi:=d g \circ \xi \circ g^{-1}$ if we consider $\xi$ as a section of the tangent bundle. If we regard $\xi$ as a derivation $\delta$ of $\mathcal{O}(X)$, then $g \delta:=\left(g^{*}\right)^{-1} \circ \delta \circ g^{*}$ where $g^{*}: \mathcal{O}(X) \rightarrow \mathcal{O}(X)$ is the comorphism of $g: X \rightarrow X$.

The action of $G$ on $\operatorname{End}(X)$ by conjugation induces a linear action on the tangent space $T_{\mathrm{id}} \operatorname{End}(X)$ which we denote by $g \mapsto \operatorname{Ad} g$. It follows that the canonical map $\Xi: T_{\text {id }} \operatorname{End}(X) \hookrightarrow \operatorname{Vec}(X)$ is $G$-equivariant. In fact, one has the formula

$$
\xi_{\operatorname{Ad} g(A)}(g x)=d g \xi_{A}(x) \text { for } A \in T_{\text {id }} \operatorname{End}(X), g \in G \text { and } x \in X .
$$

This proves the first part of the following lemma. 
Lemma 4.1. We have $\xi\left(T_{\mathrm{id}} \operatorname{End}_{G}(X)\right) \subseteq \operatorname{Vec}_{G}(X)$ with equality if $X$ is a vector space $V$ with a linear action of G.

Proof. It remains to see that for a linear action of $G$ on the vector space $V$ we have the identification $T_{\text {id }} \operatorname{End}_{G}(V)=\left(T_{\text {id }} \operatorname{End}(V)\right)^{G}$. But this is clear, because $\operatorname{End}(V)$ is a vector space, $\operatorname{End}_{G}(V)=\operatorname{End}(V)^{G}$ is a linear subspace, and $\Xi: T_{\text {id }} \operatorname{End}(V) \stackrel{\sim}{\rightarrow} \operatorname{Vec}(V)$ is a $G$-equivariant linear isomorphism.

\section{2. $\mathrm{Vec}_{G}$-symmetric subvarieties}

We now come to the main notion of this paper, the $\operatorname{Vec}_{G}$-symmetry of subvarieties. This was already discussed in the introduction.

Definition 4.2. Let $X$ be an affine variety with an action of an algebraic group $G$. A closed subvariety $Y \subseteq X$ is called $\operatorname{Vec}_{G}$-symmetric if $Y$ is $\operatorname{Vec}_{G}(X)$-invariant, i.e., $Y$ is parallel to all $G$-invariant vector fields $\xi$.

If $V$ is a vector space with a linear action of the algebraic group $G$, then $\operatorname{End}_{G}(V) \subseteq \operatorname{End}(V)$ is a

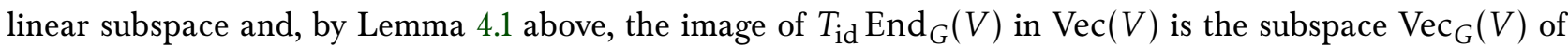
$G$-invariant vector fields. Hence Theorem 3.8 implies the following result.

Theorem 4.3. Let $V$ be a vector space with a linear action of an algebraic group $G$. Then a closed subvariety $X \subseteq V$ is $\operatorname{Vec}_{G}$-symmetric if and only if it is stable under $\operatorname{End}_{G}(V)$.

Example 4.4. Let $V$ be a $G$-module, and assume that $V^{G}=\{0\}$. Define the null cone

$$
\mathcal{N}_{0}:=\left\{v \in V \mid f(v)=0 \text { for all } f \in \mathcal{O}(V)^{G} \text { such that } f(0)=0\right\} .
$$

Then $\mathcal{N}_{0} \subseteq V$ is a closed $\operatorname{Vec}_{G}$-symmetric subvariety.

Proof. We have $\mathcal{O}(V)=\mathbb{k} \oplus \mathfrak{m}_{0}$ where $\mathfrak{m}_{0}$ is the maximal ideal of $0 \in V$, and $\mathcal{N}_{0}$ is the zero set of $\mathfrak{m}_{0}^{G}$. Since $V^{G}$ is fixed under every $G$-equivariant endomorphism $\varphi$ of $V$, we get $\varphi^{*}\left(\mathrm{~m}_{0}^{G}\right) \subseteq \mathrm{m}_{0}^{G}$, and so $\mathcal{N}_{0}$ is stable under $\operatorname{End}_{G}(V)$. Now the claim follows from the theorem above.

\subsection{Stabilizers}

The next result deals with the relation between $\operatorname{Vec}_{G}$-symmetric subvarieties and the $G$-action on $X$. We denote by $G_{x} \subseteq G$ the stabilizer of $x \in X$, and by $M(x)$ the minimal $\operatorname{Vec}_{G}(X)$-symmetric subvariety containing $x$ (Lemma 2.5(4)).

Lemma 4.5. Let $X$ be an affine G-variety.

(1) If $Y \subseteq X$ is a $\operatorname{Vec}_{G}$-symmetric closed subvariety, then $g Y \subseteq X$ is $\operatorname{Vec}_{G}$-symmetric for all $g \in G$.

(2) For $x \in X$ we have $\xi(x) \subseteq\left(T_{x} X\right)^{G_{x}}$ for all $\xi \in \operatorname{Vec}_{G}(X)$.

(3) For $x \in X$ and $g \in G$ we have $g M(x)=M(g x)$, and so $g M(x)=M(x)$ for $g \in G_{x}$.

Proof. (1) If $\xi$ is a $G$-invariant vector field, then $d g \xi(x)=\xi(g x)$ for $x \in X, g \in G$. This shows that $\xi(y) \in T_{y} Y$ if and only if $\xi(g y) \in T_{g y} g Y$, and the claim follows.

(2) The formula in (1) shows that for a $G$-invariant vector field $\xi$ we get $d g \xi(x)=\xi(x)$ for $g \in G_{x}$. Hence $\xi(x) \in\left(T_{x} X\right)^{G_{x}}$.

(3) This follows from the minimality of $M(x)$.

In case of a linear action of $G$ on a vector space $V$ we get the following result.

Proposition 4.6. Let $V$ be a G-module. 
(1) For every closed subgroup $H \subseteq G$ the fixed point set $V^{H}$ is $\operatorname{Vec}_{G}$-symmetric.

(2) For all $v \in V$ we have $M(v)=\operatorname{End}_{G}(V)(v) \subseteq V^{G_{v}}$.

Proof. (1) It is clear that $V^{H}$ is stable under all $G$-equivariant endomorphisms, and so the claim follows from Theorem 4.3.

(2) By (1), $V^{G_{v}}$ is $\operatorname{Vec}_{G}$-symmetric and contains $v$, hence $M(v) \subseteq V^{G_{v}}$ by the minimality of $M(v)$.

Example 4.7. Let $G \rightarrow \mathrm{GL}(V)$ be a diagonalizable representation of an algebraic group $G$. Then, for a generic $v \in V$, we have $\operatorname{End}_{G}(V)(v)=V$. In particular, $d_{\operatorname{End}_{G}(V)}(V)=\operatorname{dim} V$.

In fact, let $V=\bigoplus_{\chi \in \Omega} V_{\chi}$ be the decomposition into weight spaces where $\Omega \subseteq X(G)$ are those characters $\chi$ of $G$ such that $V_{\chi}:=\{v \in V \mid g v=\chi(g) \cdot v\}$ is nontrivial. Then $\operatorname{End}_{G}(V)$ contains $\mathcal{L}:=\bigoplus_{\chi \in \Omega} \mathcal{L}\left(V_{\chi}\right)$ where $\mathcal{L}(W)$ denotes the linear endomorphisms of the vector space $W$. It follows that for any $v=\left(v_{\chi}\right)_{\chi \in \Omega}$ such that $v_{\chi} \neq 0$ for all $\chi \in \Omega$ we have $\mathcal{L}(V)=V$, thus the claim.

\subsection{Reductive groups}

If $X$ is an affine $G$-variety and $Y \subseteq X$ a closed and $G$-stable subvariety, then $\operatorname{Vec}_{Y}(X) \subseteq \operatorname{Vec}(X)$ is a $G$-submodule and the linear map $\rho: \operatorname{Vec}_{Y}(X) \rightarrow \operatorname{Vec}(Y)$ is $G$-equivariant. If $Y$ is also $\operatorname{Vec}_{G}$-symmetric, then $\rho\left(\operatorname{Vec}_{G}(X)\right) \subseteq \operatorname{Vec}_{G}(Y)$. But this might be a strict inclusion, i.e., not every $G$-invariant vector field on $Y$ is obtained by restricting a $G$-invariant vector field from $X$ (see Example 6.10 in Section 6). However, if $G$ is reductive and $X$ is a vector space, then we get $\rho\left(\operatorname{Vec}_{G}(X)\right)=\operatorname{Vec}_{G}(Y)$. Indeed, $\rho: \operatorname{Vec}(X) \rightarrow \operatorname{Vec}(Y)$ is surjective and, since $G$ is reductive, maps $G$-invariants onto $G$-invariants. This gives the following result.

Lemma 4.8. Let $V$ be a $G$-module and $X \subseteq V$ a closed $G$-stable and $V e_{G}$-symmetric subvariety. If a closed subvariety $Y \subseteq X$ is $\operatorname{Vec}_{G}$-symmetric with respect to the action of $G$ on $X$, then it is also $\operatorname{Vec}_{G}-$ symmetric with respect to the action on $V$. If $G$ is reductive, then the converse also holds.

Example 4.9. Consider the adjoint representation of $\mathrm{GL}_{n}=\mathrm{GL}_{n}(\mathbb{k})$ on the matrices $\mathrm{M}_{n}=\mathrm{M}_{n}(\mathbb{k})$. It follows from classical invariant theory that $\operatorname{End}_{\mathrm{GL}_{n}}\left(\mathrm{M}_{n}\right)$ is a free module over the invariants $\mathcal{O}\left(\mathrm{M}_{n}\right)^{\mathrm{GL}_{n}}$, with basis $\left(p_{i}: A \mapsto A^{i} \mid i=0, \ldots, n-1\right)$. Note that $p_{0}$ is the constant map $A \mapsto E$. It follows that the minimal symmetric subspaces $M(A)$ are given by

$$
M(A)=\sum_{i=0}^{n-1} \mathbb{k} A^{i}
$$

In particular, a closed subset $Y \subseteq V$ is $\mathrm{GL}_{n}$-symmetric if and only if, for any $A \in Y$, the vector space spanned by all powers $A^{0}=E, A, A^{2}, \ldots$ is contained in $Y$. Note that the minimal subsets $M(A) \subseteq \mathrm{M}_{n}$ are exactly the commutative unitary subalgebras of $\mathrm{M}_{n}(\mathbb{k})$ generated by one element.

Recall that a matrix $A$ is regular if its centralizer $\left(\mathrm{GL}_{n}\right)_{A}$ has dimension $n$ which is the minimal dimension of a centralizer. Equivalently, the minimal polynomial of $A$ coincides with the characteristic polynomial of $A$. The following is known.

(1) $A$ is regular if and only if $\operatorname{dim} M(A)=n$.

(2) For a regular matrix $A$ one has $M(A)=\left(\mathrm{M}_{n}\right)^{\left(\mathrm{GL}_{n}\right)_{A}}$.

An example of a closed $\operatorname{Vec}_{G}$-symmetric subvariety is the nilpotent cone $\mathcal{N} \subseteq M_{n}$ consisting of all nilpotent matrices. It is also known that for a nilpotent matrix $N$ all powers $N^{k}$ are contained in the closure of the conjugacy class $C(N)$ of $N$, as well as their linear combinations. (In fact, $N^{\prime}:=\sum_{k \geq 0} a_{k} N^{k}$ is conjugate to $N$ if $a_{0} \neq 0$, because ker $N^{\prime j}=\operatorname{ker} N^{j}$ for all $j$.) Hence these closures $\overline{C(N)}$ are $\operatorname{Vec}_{G}$-symmetric as well.

In the example above we have $M(A)=\left(\mathrm{M}_{n}\right)^{\left(\mathrm{GL}_{n}\right)_{A}}$ for a regular matrix $A$. This is an instance of the following general result which is due to Panyushev [Pan02, Theorem 1]. For the convenience of the reader we give a short proof. 
Proposition 4.10. Let $V$ be a $G$-module where $G$ is reductive. If the closure $\overline{G v}$ of the orbit of $v$ is normal and if $\operatorname{codim}_{\overline{G v}}(\overline{G v} \backslash G v) \geq 2$, then $M(v)=V^{G_{v}}$.

Proof. The assumptions on the orbit closure imply that $\mathcal{O}(\overline{G v})=\mathcal{O}(G v)$. Let $w \in V^{G_{v}}$. We will show that there is a $G$-equivariant morphism $\varphi: V \rightarrow V$ such that $\varphi(v)=w$. Since $G_{w} \supseteq G_{v}$ there is a $G$-equivariant morphism $\mu: G v \rightarrow V$ such that $\mu(v)=w$. The comorphism has the form $\mu^{*}: \mathcal{O}(V) \rightarrow \mathcal{O}(G v)=\mathcal{O}(\overline{G v})$, hence $\mu$ extends to a morphism $\tilde{\mu}: \overline{G v} \rightarrow V$ which is again $G$-equivariant. Since $G$ is reductive and $\overline{G v} \subseteq V$ closed and $G$-stable, the morphism $\tilde{\mu}$ extends to a $G$-equivariant morphism $\varphi: V \rightarrow V$ with $\varphi(v)=w$.

\subsection{Dense orbits}

Let $X$ be an irreducible affine variety, and let $\mathcal{E} \subseteq \operatorname{End}(X)$ be a subsemigroup. An interesting question is whether $\mathcal{E}$ has a dense orbit, i.e. whether there exists an $x \in X$ such that $\overline{\mathcal{E}(x)}=X$.

Lemma 4.11. Let $\mathcal{E} \subseteq \operatorname{End}(X)$ be a linear semigroup. Then the following are equivalent.

(i) $\mathcal{E}$ has a dense orbit in $X$.

(ii) $d_{\mathcal{E}}(X)=\operatorname{dim} X$.

(iii) There exists an $x \in X$ such that $\mathcal{E}(x)=X$.

(iv) One has $\mathcal{E}(x)=X$ for all $x$ in an open dense subset of $X$.

If this holds, then $X$ is a vector space.

Proof. If $\mathcal{E}$ is a linear semigroup, then $\mathcal{E}(v) \subseteq V$ is a linear subspace and therefore closed in $X$. It is now clear that the first three statements are equivalent, and (iv) follows from (iii) and the last statement of Lemma 3.5.

Proposition 4.12. Let $G$ be a reductive group, and let $V$ be a faithful $G$-module.

(1) If the generic G-orbits in $V$ are closed with trivial stabilizer, then $\operatorname{End}_{G}(V)$ has a dense orbit in $V$, i.e. $d_{\operatorname{End}_{G}(V)}(V)=\operatorname{dim} V$.

(2) If $G$ is semisimple and $d_{\operatorname{End}_{G}(V)}(V)=\operatorname{dim} V$, then the generic $G$-orbits in $V$ are closed with trivial stabilizer.

Proof. Set $\mathcal{E}:=\operatorname{End}_{G}(V)$.

(1) If the orbit $G v$ is closed and $G_{v}$ trivial, then $\mathcal{E}(v)=V$ by Proposition 4.10.

(2) If $d_{\mathcal{E}}(V)=\operatorname{dim} V$, then, by the lemma above, we have $\mathcal{E}(v)=V$ for all $v$ from a dense open subset $U \subseteq V$. Since $\mathcal{E}(v) \subseteq V^{G_{v}}$ and since the action is faithful, we see that $G_{v}$ is trivial for all $v \in U$, i.e. the generic stabilizer is trivial. Since $G$ is semisimple this implies that the generic orbits are closed, see [Pop70, Corollary 1].

Remark 4.13. Example 4.7 shows that the assumption in (2) that $G$ is semisimple is necessary.

\subsection{Invariance of the isotropy strata}

Let $G$ be a reductive group acting on an affine variety $X$, and let $\pi: X \rightarrow Z:=X / / G$ denote the algebraic quotient, i.e. the morphism corresponding to the inclusion $\mathcal{O}(X)^{G} \hookrightarrow \mathcal{O}(X)$. Then $\pi$ sets up a bijection between the closed orbits of $X$ and the points of $X / / G$.

Let $x \in X$ be such that the orbit $G x$ is closed. Then the isotropy group $H:=G_{x}$ is reductive. The isotropy stratum $Z_{H} \subseteq Z$ consists of the closed orbits whose isotropy groups are conjugate to the reductive subgroup $H \subseteq G$.

If $X$ is a $G$-module $V$ with quotient $Y:=V / / G$, then the isotropy strata are locally closed and irreducible. In fact, $Y_{H}$ is open (and dense) in the closed subset $\pi\left(V^{H}\right) \subseteq Y$, since it is equal to $\pi\left(V^{H}\right) \backslash \bigcup_{L \supsetneq H} Y_{L}$. 
Proposition 4.14. Assume that the canonical map $p_{X}: \operatorname{Vec}_{G}(X) \rightarrow \operatorname{Vec}(X / / G)$ is surjective. Then the isotropy strata of the algebraic quotient $X / / G$ are stable under the connected component $\operatorname{Aut}(X / / G)^{\circ}$ of the automorphism group of $X / / G$.

Proof. (1) We first consider the case where $X$ is a $G$-module $V$ with quotient $Y:=V / / G$. It is clear that a $G$-equivariant endomorphism $\varphi: V \rightarrow V$ sends $V^{H}$ to $V^{H}$, and thus induces an endomorphism $\bar{\varphi}$ of the quotient $V / / G$ such that $\bar{\varphi}\left(\overline{Y_{H}}\right) \subseteq \overline{Y_{H}}$. It follows that these closures are $\operatorname{End}_{G}(V)$-stable, hence invariant under $\operatorname{Vec}_{G}(V)$, by Proposition 3.3 and Lemma 4.1.

If the canonical map $p_{V}: \operatorname{Vec}_{G}(V) \rightarrow \operatorname{Vec}(Y)$ is surjective, then the closures of the strata $Y_{H}$ are invariant under $\operatorname{Vec}(Y)$. Since Lie $\operatorname{Aut}(Y)$ is a Lie-subalgebra of $\operatorname{Vec}(Y)$, it follows from [FK18, Proposition 7.2.6] that the closures $\overline{Y_{H}}$ are stable under $\operatorname{Aut}(Y)^{\circ}$. Since the closure of every stratum is a finite union of strata we finally get that the strata $Z_{H}$ are $\operatorname{Aut}(Z)^{\circ}$-stable.

(2) In general, we can assume that $Z$ is a $G$-stable closed subset of a $G$-module $V$, and so $Z:=X / / G$ is a closed subset of $Y:=V / / G$. By definition, the isotropy strata of $Z$ are the intersections $Z_{H}=Y_{H} \cap Z$. We have the following commutative diagram where the horizontal maps are the restriction maps of vector fields to closed subsets:

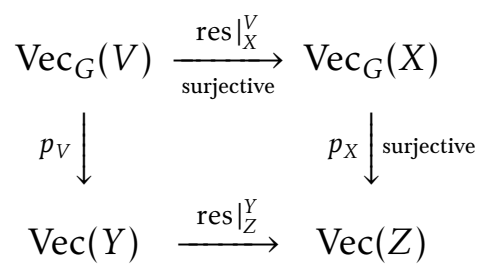

Since $V$ is a vector space, the restriction $\operatorname{map} \operatorname{Vec}(V) \rightarrow \operatorname{Vec}(X)$ is surjective, hence res $\left.\right|_{X} ^{V}$ is also surjective, because $G$ is reductive. By assumption, $p_{X}$ is also surjective.

We have seen in (1) that the closures of the isotropy strata of $Y$ are invariant under the image of $\operatorname{Vec}_{G}(V)$ in $\operatorname{Vec}(Y)$. Hence, the closures of the isotropy strata of $Z$ are invariant under the image of $\operatorname{Vec}_{G}(V)$ in $\operatorname{Vec}(Z)$ which is all of $\operatorname{Vec}(Z)$ as we have just seen. Now the claim follows as in (1).

Remark 4.15. Proposition 4.14 is a variant of a stronger result of Schwarz [Sch13] (cf. Remark 3.11) which shows that under the same assumptions the (irreducible) Luna-strata are permuted under the full automorphism group of the quotient $X / / G$. In his proof he shows that the vector fields span the tangent spaces of the Luna-strata, and thus an automorphism has to permute the strata of the same dimension.

\section{First integrals}

\subsection{The field of first integrals}

Let $X$ be an irreducible affine variety, and let $\mathcal{D} \subseteq \operatorname{Vec}(X)$ be a linear subspace.

Definition 5.1. A first integral of $\mathcal{D}$ is a rational function $f \in \mathbb{k}(X)$ with the property that $\xi f=0$ for all $\xi \in \mathcal{D}$. If $X$ is a $G$-variety and $\mathcal{D}:=\operatorname{Vec}_{G}(X)$, then a first integral of $\mathcal{D}$ will be called a first integral for the $G$-action on $X$.

It is easy to see that the first integrals of $\mathcal{D}$ form a subfield of $\mathrm{k}(X)$ which we denote by $\mathcal{F}_{\mathcal{D}}(X)$. If $\mathcal{D}=\operatorname{Vec}_{G}(X)$, then we write $\mathcal{F}_{G}(X)$ instead of $\mathcal{F}_{\operatorname{Vec}_{G}(X)}(X)$.

From now on assume that $X$ is an irreducible affine variety, and that $\mathcal{D} \subseteq \operatorname{Vec}(X)$ is a linear subspace. We want to show that the first integrals are the rational functions on a certain "quotient" of the variety $X$ which will be defined for the action of a linear semigroup $\mathcal{E} \subseteq \operatorname{End}(X)$ in a similar way as the quotient for the action of an algebraic group, see Section 5.2. 
Lemma 5.2. Let $f \in \mathbb{k}(X)$ be a rational function.

(1) Assume that there is an open dense $U \subseteq X$ where $f$ is defined and has the property that $f$ is constant on $M(x) \cap U$ for all $x \in U$. Then $f$ is a first integral of $\mathcal{D}$.

(2) Assume that $f$ is a first integral of $\mathcal{D}$. If $f$ is defined in $x \in X$ and if $T_{x} M(x)=\mathcal{D}(x)$, then $f$ is constant on $M(x)$.

Proof. (1) Since $M(x)$ is $\mathcal{D}$-invariant we have $\xi(x) \in T_{x} M(x)$ for all $x \in U$ and all $\xi \in \mathcal{D}$. Hence we have $(\xi f)(x)=\xi(x) f=\left.\xi(x) f\right|_{M(x) \cap U}=0$, because $\left.f\right|_{M(x) \cap U}$ is constant, and so $\xi f=0$ for all $\xi \in \mathcal{D}$.

(2) There is $d \geq 0$ such that $\operatorname{dim} \mathcal{D}(y) \leq d$ for all $y \in M(x)$, with equality on a dense open set $M^{\prime} \subseteq M(x)$ (Lemma 2.7). In particular, $\operatorname{dim} M(x) \leq \operatorname{dim} T_{x} M(x)=\operatorname{dim} \mathcal{D}_{x} \leq d$. On the other hand, $\mathcal{D}_{y} \subseteq T_{y} M(x)$ for all $y \in M(x)$. We can assume that $M^{\prime}$ consists of smooth point of $M(x)$. Then, for every $y \in M^{\prime}$, we get $d=\operatorname{dim} \mathcal{D}_{y} \leq \operatorname{dim} T_{y} M(x)=\operatorname{dim} M(x)$. Hence $d=\operatorname{dim} M(x)$, and so $T_{y} M(x)=\mathcal{D}(y)$ for all $y \in M^{\prime}$. Since $f$ is defined in $x$, it is defined in a dense open set $M^{\prime \prime} \subseteq M^{\prime}$. But then $\left.f\right|_{M^{\prime \prime}}$ is constant, because $\delta f=0$ for all $u \in M^{\prime \prime}$ and all $\delta \in T_{u} M(x)$.

Remark 5.3. If $\mathcal{E} \subseteq \operatorname{End}(X)$ is a linear semigroup and $\mathcal{D}:=\mathcal{D}_{\mathcal{E}}$, then a rational function $f \in \mathbb{k}(X)$ defined on an open set $U \subseteq X$ is a first integral for $\mathcal{D}$ if and only if $f$ is constant on $\mathcal{E}(x) \cap U$ for all $x \in U$. This follows from the lemma above, because in this case we have $\mathcal{E}(x)=M(x)$ and $T_{x} M(x)=\mathcal{D}(x)$ for all $x \in X$, by Theorem 3.8.

Now choose a closed embedding $X \subseteq V$ into a vector space $V$. We know from Lemma 2.7 that the subset $X^{\prime}:=\left\{x \in X \mid \operatorname{dim} \mathcal{D}(x)=d_{\mathcal{D}}(x)\right\}$ is open and dense in $X$. Consider the map

$$
\pi: X^{\prime} \rightarrow \operatorname{Gr}_{d_{\mathcal{D}}(X)}(V) \text { given by } \pi(x):=\mathcal{D}(x) \subseteq T_{x} X \subseteq V .
$$

Lemma 5.4. The map $\pi: X^{\prime} \rightarrow \mathrm{Gr}_{d_{\mathcal{D}}(x)}(V)$ is a morphism of varieties.

Proof. We will use the Plücker-embedding $\operatorname{Gr}_{d}(V) \hookrightarrow \mathbb{P}\left(\bigwedge^{d} V\right), d:=d_{\mathcal{D}}(x)$. For $x \in X^{\prime}$ choose $\xi_{1}, \ldots, \xi_{d} \in \mathcal{D}$ such that $\xi_{1}(x), \ldots, \xi_{d}(x)$ is a basis of $\mathcal{D}(x)$. Then $\mathcal{D}(x)=\xi_{1}(x) \wedge \xi_{2}(x) \wedge \cdots \wedge \xi_{d}(x) \in \wedge^{d} V$. It follows that there is an open neighborhood $U_{x} \subseteq X^{\prime}$ of $x$ such that $\xi_{1}(u), \ldots, \xi_{d}(u)$ is a basis of $\mathcal{D}(u)$ for all $u \in U_{x}$. Since $\pi(u)=\left[\xi_{1}(u) \wedge \cdots \wedge \xi_{d}(u)\right] \in \mathbb{P}\left(\wedge^{d} V\right)$ we see that $\left.\pi\right|_{U_{x}}$ is a morphism, and the claim follows.

\subsection{The quotient $\bmod \mathcal{E}$}

Let $\mathcal{E} \subseteq \operatorname{End}(V)$ be a linear semigroup, and let $\mathcal{D}_{\mathcal{E}} \subseteq \operatorname{Vec}(V)$ denote the image of $T_{\text {id }} \mathcal{E}=\mathcal{E}$. Let $X \subseteq V$ be a closed irreducible $\mathcal{E}$-stable subvariety. Under these assumptions we have $\mathcal{E}(x)=M(x)=\mathcal{D}_{\mathcal{E}}(x) \subseteq V$ for all $x \in X$ (Lemma 3.5). As above, define

$$
X^{\prime}:=\left\{x \in X \mid \operatorname{dim} \mathcal{E}(x)=d_{\mathcal{E}}(X)\right\},
$$

and consider the morphism $\pi: X^{\prime} \rightarrow \mathrm{Gr}_{d_{\mathcal{E}}(x)}(V), x \mapsto \mathcal{E}(x) \subseteq T_{x} X \subseteq V$.

\section{Proposition 5.5.}

(1) For all $x \in X^{\prime}$ we have $\pi^{-1}(\pi(x))=\mathcal{E}(x) \cap X^{\prime}$.

(2) $\pi$ induces an isomorphism $\pi^{*}: \mathbb{k}\left(\overline{\pi\left(X^{\prime}\right)}\right) \stackrel{\sim}{\rightarrow} \mathcal{F}_{\mathcal{D}_{\mathcal{E}}}(X)$.

(3) We have $\operatorname{tdeg} \mathcal{F}_{\mathbb{R}} \mathcal{F}_{\mathcal{E}}(X)=\operatorname{dim} X-d_{\mathcal{E}}(X)=\operatorname{dim} \overline{\pi\left(X^{\prime}\right)}$.

(4) $\mathcal{F}_{\mathcal{D}_{\mathcal{E}}}(X)=\mathbb{k}$ if and only if $d_{\mathcal{E}}(X)=\operatorname{dim} X$, and then $X \subseteq V$ is a linear subspace.

The proposition shows that the orbits on the open subvariety $X^{\prime} \subseteq X$, i.e. the subsets $\mathcal{E}(x) \cap X^{\prime}$, are disjoint and are the fibers of the morphism $\pi: X^{\prime} \rightarrow \operatorname{Gr}_{d_{\mathcal{E}}(x)}(V)$. Therefore, we will use the notion $X / / \mathcal{E}$ for the closure $\overline{\pi\left(X^{\prime}\right)}$ and call it the quotient of $X$ under the action of the semigroup $\mathcal{E}$ of endomorphisms. 
Proof. (1) For $y \in \mathcal{E}(x) \cap X^{\prime}$ we have $\mathcal{E}(y)=\mathcal{E}(x)$, hence $\pi(y)=\pi(x)$. If $y \in X^{\prime} \backslash \mathcal{E}(x)$, then $\mathcal{E}(y) \neq \mathcal{E}(x)$ and so $\pi(y) \neq \pi(x)$.

(2) By Remark 5.3 a rational function $f \in \mathbb{k}(X)$ defined on an open set $U \subseteq X^{\prime}$ is a first integral if and only if it is constant on the subsets $\mathcal{E}(x) \cap U$ for all $x \in U$. We can assume that $\pi(U) \subseteq \mathrm{Gr}_{d_{\mathcal{E}}(x)}(V)$ is locally closed and smooth and that $\pi: U \rightarrow \pi(U)$ is smooth. Then it is a well-known fact that $\pi^{*}(\mathcal{O}(\pi(U))) \subseteq \mathcal{O}(U)$ are the regular functions on $U$ which are constant on the fibers.

(3) This is clear.

(4) If $d_{\mathcal{E}}(X)=\operatorname{dim} X$, then $X=\mathcal{E}(x)$ for a generic $x \in X$ (Lemma 4.11), and so $X$ is a linear subspace of $V$.

Corollary 5.6. If $X \subseteq V$ is not a linear subspace, then there exist non-constant first integrals.

Note that if $X$ is smooth, then it is a linear subspace, because $X$ is a closed cone, see Remark 3.6.

Example 5.7. Let $X \subseteq V$ be a closed cone, and let $\mathcal{E}:=\mathbb{k} \cdot \operatorname{id}_{V} \subseteq \operatorname{End}(V)$. Then $\mathcal{E}(x)=\mathbb{k} x$ for all $x \in X$, hence $X / / \mathcal{E}=\mathbb{P}(X)$ and $\mathcal{F}_{\mathcal{D}_{\mathcal{E}}}(X)=\mathbb{k}(\mathbb{P}(X))$.

\subsection{The symmetric case}

Assume that $V$ is a representation of an algebraic group and that $\mathcal{E}:=\operatorname{End}_{G}(V)$, hence $\mathcal{D}_{\mathcal{E}}=\operatorname{Vec}_{G}(V)$. Then, for every $G$-stable and $\operatorname{Vec}_{G}$-symmetric closed irreducible subvariety $X \subseteq V$, the open subset $X^{\prime} \subseteq X$ is $G$-stable and the morphism $\pi: X^{\prime} \rightarrow \mathrm{Gr}_{d_{\mathcal{E}}(x)}(V)$ is $G$-equivariant. In particular, $\pi^{*}: \mathbb{k}\left(\overline{\pi\left(X^{\prime}\right)}\right) \stackrel{\sim}{\rightarrow} \mathcal{F}_{G}(X)$ is a $G$-equivariant isomorphism. It follows that for any $x \in X^{\prime}$ we have

$$
G_{\pi(x)}=\operatorname{Norm}_{G}(\mathcal{E}(x))
$$

where $\operatorname{Norm}_{G}(W)$ denotes the normalizer in $G$ of the subspace $W \subseteq V$.

\section{Lemma 5.8.}

(1) For $x \in X^{\prime}$ we have

$$
\operatorname{tdeg} \mathcal{F}_{G}(X) \geq \operatorname{tdeg} \mathcal{F}_{G}(X)^{G}+\operatorname{dim} G-\operatorname{dim} \operatorname{Norm}_{G}(\mathcal{E}(x))
$$

with equality on a dense open set $U \subseteq X^{\prime}$.

(2) If $\mathcal{F}_{G}(X)^{G}=\mathbb{k}$, then $\mathcal{F}_{G}(X)$ is $G$-isomorphic to $\mathbb{k}\left(G / \operatorname{Norm}_{G}(\mathcal{E}(x))\right.$ for any $x$ in a dense open set of $X^{\prime}$.

Proof. (1) By Rosenlicht's theorem (see [Spr89, Satz 2.2]) there is an open dense G-stable subset $O \subseteq \pi\left(X^{\prime}\right)$ which admits a geometric quotient $q: O \rightarrow O / G$. In particular, the fibers of $q$ are $G$-orbits and have all the same dimension. Hence $\operatorname{tdeg} \mathcal{F}_{G}(X)=\operatorname{dim} O=\operatorname{dim} O / G+\operatorname{dim} G u$ for $u \in O$, and we also have the equality $\mathbb{k}(O / G)=\mathbb{k}(O)^{G}=\mathcal{F}_{G}(X)^{G}$. If $u=\pi(x)$, then $G_{u}=\operatorname{Norm}_{G}(\mathcal{E}(x))$ and so

$$
\operatorname{tdeg} \mathcal{F}_{G}(X)=\operatorname{tdeg} \mathcal{F}_{G}(X)^{G}+\operatorname{dim} G-\operatorname{dim} \operatorname{Norm}_{G}(\mathcal{E}(x))
$$

for all $x \in U:=\pi^{-1}(O)$. Since $\operatorname{dim} G u$ is maximal for $u \in O$ the claim follows.

(2) If $\mathcal{F}_{G}(X)^{G}=\mathbb{k}$, then, as a consequence of Rosenlicht's theorem, $G$ has a dense orbit $G u$ in $\overline{\pi\left(X^{\prime}\right)}$ and so $\mathcal{F}_{G}(X)=\mathbb{k}(G u)$. If $u=\pi(x)$, then $G u \simeq G / \operatorname{Norm}_{G}(\mathcal{E}(x))$, and the claim follows.

Remark 5.9. Note that $\mathcal{F}_{G}(X)^{G}=\mathbb{k}$ if and only if $G \mathcal{E}(x)$ is dense in $X$ for a generic $x \in X$, or, equivalently, $\operatorname{dim} X=d_{\mathcal{E}}(x)+\operatorname{dim} G-\operatorname{dim} \operatorname{Norm}_{G}(\mathcal{E}(x))$ for a generic $x \in X$.

Example 5.10. Consider the adjoint representation of $\mathrm{GL}_{2}$ on $\mathrm{M}_{2}$. Then $\mathrm{M}_{2}^{\prime}=\mathrm{M}_{2} \backslash \mathbb{k} I_{2}$ where $I_{2}=\left[\begin{array}{ll}1 & 0 \\ 0 & 1\end{array}\right]$, and the morphism $\pi$ is equal to the composition

$$
\pi: \mathrm{M}_{2}^{\prime} \rightarrow\left(\mathrm{M}_{2} / \mathbb{k} I_{2}\right) \backslash\{0\} \rightarrow \mathbb{P}\left(\mathrm{M}_{2} / \mathbb{k} I_{2}\right) .
$$




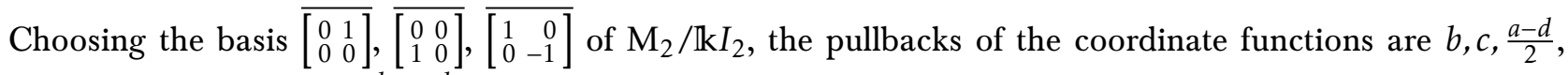
and so $\mathcal{F}_{\mathrm{GL}_{2}}\left(\mathrm{M}_{2}\right)=\mathbb{k}\left(\frac{a-d}{b}, \frac{a-d}{c}\right)$.

Example 5.11. For the adjoint representation of $\mathrm{GL}_{n}$ on $\mathrm{M}_{n}$ we claim that $\mathrm{GL}_{n}$ has a dense orbit in $\pi\left(\mathrm{M}_{n}^{\prime}\right)$. In fact, let $S \in \mathrm{M}_{n}$ be a generic diagonal matrix. Then the span $\mathcal{E}(S)=\sum_{i=0}^{n-1} \mathrm{k} S^{i}$ has dimension $n$, hence it is the subspace of diagonal matrices, and so $\mathrm{GL}_{n} \mathcal{E}(S) \subseteq \mathrm{M}_{n}$ is the dense subset of all diagonalizable matrices. Moreover, the normalizer of $\mathcal{E}(S)$ is equal to $N$, the normalizer of the diagonal torus $T \subseteq \mathrm{GL}_{n}$, and so $\mathcal{F}_{\mathrm{GL}_{n}}\left(\mathrm{M}_{n}\right) \simeq \mathbb{k}\left(\mathrm{GL}_{n} / N\right)$.

Example 5.12. The previous example carries over to the adjoint representation of an arbitrary semisimple group $G$ on its Lie algebra $\mathfrak{g}:=\operatorname{Lie} G$. If $s \in \mathfrak{g}$ is a regular semisimple element, then the orbit $G s$ is closed and the stabilizer of $s$ is a maximal torus $T$. This implies by Proposition 4.10 that $\mathcal{E}(s)=\mathfrak{g}^{T}=\operatorname{Lie} T$ which is a Cartan subalgebra of $\mathfrak{g}$. Again, $G \mathcal{E}(s) \subseteq \mathfrak{g}$ is the dense set of semisimple elements of $\mathfrak{g}$, and the normalizer of $\mathcal{E}(s)$ is equal to $N$, the normalizer of $T$ in $G$. Hence $\mathcal{F}_{G}($ Lie $G) \simeq \mathbb{k}(G / N)$.

In the examples above there are no $G$-invariant first integrals: $\mathcal{F}_{G}(X)^{G}=\mathbb{k}$. This is not always the case as the next two examples show. However, it holds for a representation of a reductive group $G$ in case the generic fiber of the quotient map contains a dense orbit (Proposition 5.17).

Example 5.13. Suppose that $U \subseteq \mathrm{GL}(V)$ is unipotent and that the generic stabilizer of the action of $U$ on $V$ is trivial. Then it follows from a result of Domokos [Dom08, Theorem 1.1, p.840] that $\mathcal{F}_{U}(V) \simeq \mathbb{k}$. In this example we look at an instance where the generic stabilizer is not trivial.

Let $U=\left\{\left[\begin{array}{ccc}1 & a & b \\ 1 & c \\ & 1\end{array}\right] \mid a, b, c \in \mathbb{k}\right\} \subseteq \mathrm{GL}_{3}(\mathbb{k})$ be the unipotent group of upper triangular matrices, and consider the adjoint representation of $U$ on its Lie algebra $\mathfrak{u}:=\operatorname{Lie} U=\left\{\left[\begin{array}{ccc}0 & y \\ 0 & z \\ & 0\end{array}\right] \mid x, y, z \in \mathbb{k}\right\}$. For $u=\left[\begin{array}{ccc}1 & a & b \\ 1 & c \\ 1\end{array}\right] \in$ $U$ and $v=\left[\begin{array}{rr}0 & x \\ 0 & z \\ & 0\end{array}\right] \in \mathfrak{u}$ we find

$$
\operatorname{Ad}(u) v=u v u^{-1}=\left[\begin{array}{ccc}
0 & x & -c x+y+a z \\
& 0 & z \\
& & 0
\end{array}\right]
$$

which shows that the fixed points are $\mathfrak{u}^{U}=\mathbb{k}\left[\begin{array}{ccc}0 & 0 & 1 \\ 0 & 0 \\ 0\end{array}\right]$ and the other orbits are the parallel lines $\operatorname{Ad}(U)\left[\begin{array}{rrr}0 & x & y \\ 0 & z \\ & 0\end{array}\right]=\left[\begin{array}{ccc}0 & x & 0 \\ 0 & z \\ 0\end{array}\right]+\mathfrak{u}^{U}$. It follows that the invariant ring is given by $\mathcal{O}(\mathfrak{u})^{U}=\mathbb{k}[x, z]$. We have an exact sequence of $U$-modules

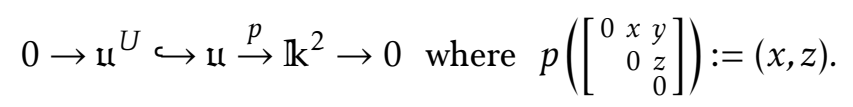

We claim that the covariants $\mathcal{E}:=\operatorname{Cov}(\mathfrak{u}, \mathfrak{u})$ are generated as a $\mathcal{O}(\mathfrak{u})^{U}$-module by $\mathrm{id}_{\mathfrak{u}}$ and the map

$$
\varphi_{0}:\left[\begin{array}{rrr}
0 & x & y \\
0 & z \\
0 & 0
\end{array}\right] \mapsto\left[\begin{array}{ccc}
0 & 0 & 1 \\
0 & 0 \\
& 0
\end{array}\right] \text {. }
$$

This implies that $\mathcal{E}(v)=\mathbb{k} v+\mathfrak{u}^{U}$ for $v \in \mathfrak{u} \backslash \mathfrak{u}^{U}$, hence $d_{\mathcal{E}}(\mathfrak{u})=2$ and $\mathfrak{u}^{\prime}=\mathfrak{u} \backslash \mathfrak{u}^{U}$. It follows that

$$
\mathfrak{u} / / \mathcal{E}=\mathbb{P}\left(\mathfrak{u} / \mathfrak{u}^{U}\right) \stackrel{\sim}{\rightarrow} \mathbb{P}^{1} .
$$

In particular, the action of $U$ on the quotient is trivial, and so

$$
\mathcal{F}_{U}(\mathfrak{u})=\mathcal{F}_{U}(\mathfrak{u})^{U}=\mathbb{k}\left(\frac{x}{z}\right) .
$$

In order to prove the claim, let $\varphi: \mathfrak{u} \rightarrow \mathfrak{u}$ be a covariant,

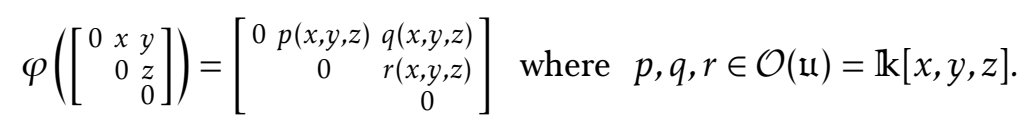


Then, by (**), we get for $a, b, c \in \mathbb{k}$

$$
q(x,-c x+y+a z, z)=-c \cdot p(x, y, z)+q(x, y, z)+a \cdot r(x, y, z) .
$$

This shows that $q$ is linear in $y$, i.e. $q(x, y, z)=q_{0}(x, z)+q_{1}(x, z) y$, and so

$$
q(x,-c x+y+a z, z)=q_{0}(x, y)+q_{1}(x, z)(-c x+y+a z)=q_{0}-c \cdot q_{1} x+q_{1} y+a \cdot q_{1} z
$$

Comparing (2) with (1) we get

$$
p=q_{1} x, \quad q=q_{0}+q_{1} y, \quad r=q_{1} z,
$$

hence $\varphi=q_{1} \mathrm{id}_{\mathfrak{u l}}+q_{0} \varphi_{0}$, as claimed.

Example 5.14. Let $G$ be a reductive group and $V$ an irreducible $G$-module. If the connected component of the center $Z(G)^{0}$ acts nontrivially, then $\operatorname{End}_{G}(V)=\mathbb{k i d}_{V}$. Hence, by Example 5.7, we get the following equalities $V / / \operatorname{End}_{G}(V) \simeq \mathbb{P}(V), \mathcal{F}_{G}(V)=\mathbb{k}(\mathbb{P}(V))$, and $\mathcal{F}_{G}(V)^{G}=\mathbb{k}(\mathbb{P}(V / /(G, G)))$.

(In order to see that $\operatorname{End}_{G}(V)=\mathrm{kid}_{V}$ we just remark that the $G$-module $V^{*}$ occurs only once in $\mathcal{O}(V)$, namely in degree 1 . In fact, $Z(G)^{0}$ acts on $V$ via a character $\chi$, and thus via $\chi^{-d}$ on the homogeneous functions $\mathcal{O}(V)_{d}$ of degree $d$.)

This example generalizes to the situation where $V$ is a reducible $G$-module such that the characters of $Z(G)^{0}$ on the irreducible components of $V$ are linearly independent.

Example 5.15. Let $V$ be an irreducible representation of a reductive group $G$. For the orbit $O_{\text {min }} \subseteq V$ of highest weight vectors we have $\overline{O_{\min }}=O_{\min } \cup\{0\}$, and $\overline{O_{\min }}$ is normal with rational singularities (see [Hes79]). Clearly, $\overline{O_{\min }}$ is $\operatorname{Vec}_{G}$-symmetric, i.e. stable under all $G$-equivariant endomorphisms of $V$. We claim that $\mathcal{E}:=\operatorname{End}_{G}\left(\overline{O_{\text {min }}}\right)=\mathbb{k} \cdot \mathrm{id}$. In fact, if $v \in V$ is a highest weight vector, then the $G$-orbit of $[v] \in \mathbb{P}(V)$ is closed, and thus the stabilizer $P$ of $[v]$ is a parabolic subgroup. Hence $P$ is the normalizer of $G_{v}$ in $G$, and so $P / G_{v}=\mathbb{k}^{*}$. Since, Aut ${ }_{G}\left(\overline{O_{\text {min }}}\right)=\operatorname{Aut}_{G}\left(O_{\text {min }}\right) \simeq P / G_{v}=\mathbb{k}^{*}$ the claim follows.

As a consequence we get $\overline{O_{\text {min }}}=O_{\text {min }}, \overline{O_{\text {min }}} / / \mathcal{E}=O_{\text {min }} / \mathbb{k}^{*}=\mathbb{P}\left(\overline{O_{\text {min }}}\right) \subseteq \mathbb{P}(V)$, and so $\mathbb{P}\left(\overline{O_{\text {min }}}\right)$ is the closed orbit of highest weight vectors in $\mathbb{P}(V)$. In particular, $\mathcal{F}_{G}\left(\overline{O_{\text {min }}}\right)=\mathbb{k}\left(\mathbb{P}\left(O_{\text {min }}\right)\right)$, and $\mathcal{F}_{G}\left(\overline{O_{\text {min }}}\right)^{G}=\mathbb{k}$.

\subsection{First integrals for reductive groups}

Let $G$ be a reductive group, and let $X$ be an irreducible $G$-variety. Denote by $q: X \rightarrow X / / G$ the quotient. Then Luna's slice theorem (see [Lun73, pp. 97-98]) implies the existence of a principal isotropy group $H \subseteq G$. This means the following:

(1) If $G x \subseteq X$ is a closed orbit, then $G_{x}$ contains a conjugate of $H$.

(2) The set $(X / / G)_{p r}$ of points $\xi \in X / / G$ such that the closed orbit in the fiber $q^{-1}(\xi)$ is $G$-isomorphic to $G / H$ is open and dense in $X / / G$.

It follows that every closed orbit contains a fixed point of $H$, hence $\pi\left(X^{H}\right)=X / / G$.

The open dense subset $(X / / G)_{\mathrm{pr}}$ of $X / / G$ is called the principal stratum, and the closed orbits over the principal stratum are the principal orbits. If the action on $X$ is stable, i.e. if the generic orbits of $X$ are closed, then the principal orbits are generic.

Theorem 5.16. Let $G$ be reductive, $V$ a $G$-module, and let $X \subseteq V$ be $a G$-stable and $V \mathrm{Vec}_{G}$-symmetric irreducible closed subvariety. Assume that the generic orbit of $X$ is closed, with principal isotropy group $H \subseteq G$. Then $\mathcal{F}_{G}(X)=\mathbb{k}(G / N)$ where $N:=\operatorname{Norm}_{G}(H)$. In particular, $\mathcal{F}_{G}(X)^{G}=\mathbb{k}$.

Proof. By assumption, the orbit $G x$ is principal for a generic $x \in X^{H}$. The minimal invariant subset $M(x)$ of $X$ is also minimal invariant as a subset of $V$ (Lemma 4.8). Hence, $M(x)=V^{H}$ by Proposition 4.10. Since $M(x) \subseteq X^{H} \subseteq V^{H}$, we finally get $M(x)=X^{H}=V^{H}$. As we have seen above, $G X^{H}$ contains all closed orbits, 
and in particular all $M(y)$ for $y$ in the dense open set of principal orbits. This implies that $G$ has a dense orbit in $X / / \operatorname{End}_{G}(X)$. Since the stabilizer of the image $\pi\left(V^{H}\right)$ is the normalizer $\operatorname{Norm}_{G}\left(V^{H}\right)$, it remains to see $\operatorname{Norm}_{G}\left(V^{H}\right)=\operatorname{Norm}_{G}(H)$. Since $g\left(V^{H}\right)=V^{g H g^{-1}}$ we get $V^{H}=V^{H \cap g H g^{-1}}$ for any $g \in \operatorname{Norm}\left(V^{H}\right)$, hence $H=g \mathrm{Hg}^{-1}$, because the stabilizer of a generic elements from $V^{H}$ is $H$.

Note that for a "generic" representation of a semisimple group $G$ the principal isotropy group is trivial, hence there are no non-constant $G$-invariant first integrals. The irreducible representations of simple groups with a nontrivial principal isotropy group have been classified ([AVE67], [Pop75], cf. [PV94, §7]).

The fact that there are no non-constant $G$-invariant first integrals is a consequence of the following slightly more general result.

Proposition 5.17. Let $V$ be a representation of a reductive group $G$. Assume that the generic fiber of the quotient map $q: V \rightarrow V / / G$ contains a dense orbit $O \simeq G / K$, i.e. $\mathbb{k}(V)^{G}$ is the field of fractions of $\mathcal{O}(V)^{G}$, and that $\operatorname{codim}_{F} F \backslash O \geq 2$. Then $\mathcal{F}_{G}(V) \simeq \mathbb{k}\left(G / \operatorname{Norm}_{G}(K)\right)$ and $\mathcal{F}_{G}(V)^{G}=\mathbb{k}$.

Proof. Let $F$ be a fiber of the quotient map $q$ over the principal stratum, and let $O \subseteq F$ be the dense orbit. Consider the morphism $\pi: V^{\prime} \rightarrow V / / \operatorname{End}_{G}(V) \subseteq \operatorname{Gr}_{d}(V), d:=d_{\operatorname{End}_{G}(V)}(V)$. We claim that $O \subseteq V^{\prime}$, that $\overline{\pi(O)}=\overline{\pi\left(V^{\prime}\right)}=V / / \operatorname{End}_{G}(V)$, and that the image of $O$ under $\pi$ is $G / \operatorname{Norm}_{G}(K)$. This will prove the proposition.

Luna's slice theorem tells us that all the fibers of the quotient map $q$ over the principal stratum are $G$-isomorphic. This implies that $\operatorname{End}_{G}(V)$ acts transitively on the set of these fibers (see the argument in the proof of Proposition 4.10), hence $\overline{\pi\left(V^{\prime}\right)}=\overline{\pi\left(F \cap V^{\prime}\right)}$. Since $F \cap V^{\prime}$ is open and $G$-stable, we have $O \subseteq V^{\prime}$. If $\varphi \in \operatorname{End}_{G}(V)$ and $\varphi(v) \in O$ for some $v \in O$, then $\varphi(O)=O$, and so $\left.\varphi\right|_{O}$ is a $G$-equivariant automorphism. On the other hand, let $\psi: O \rightarrow O$ be a $G$-equivariant automorphism. Since $F$ is smooth and the complement of the orbit $O \subseteq F$ has codimension $\geq 2$ we have $\mathcal{O}(O)=\mathcal{O}(F)$. Therefore, $\psi$ extends to a $G$-equivariant automorphism of $F$, and then lifts to a $G$-equivariant endomorphism of $V$. This implies that $\operatorname{End}_{G}(V) v \cap O=\operatorname{Aut}_{G}(O) v$. Hence, $\pi(O) \simeq O / \operatorname{Aut}_{G}(O) \simeq G / \operatorname{Norm}_{G}(K)$, and the claims follow.

\section{Actions of $\mathrm{SL}_{2}$}

\subsection{Representations}

The standard representation of $\mathrm{SL}_{2}$ on $V:=\mathbb{k}^{2}$ defines a linear action given by $g f(v):=f\left(g^{-1} v\right)$ on the coordinate ring $\mathcal{O}(V)=\mathbb{k}[x, y]$. It is well-known that the homogeneous components $V_{d}:=\mathbb{k}[x, y]_{d}$, $d=0,1,2, \ldots$, represent all irreducible representations of $\mathrm{SL}_{2}$, i.e. all simple $\mathrm{SL}_{2}$-modules. As usual, $B \subseteq \mathrm{SL}_{2}$ denotes the Borel-subgroup of upper triangular matrices, $T \subseteq \mathrm{SL}_{2}$ the diagonal torus, and $N \subseteq \mathrm{SL}_{2}$ the normalizer of $T$.

Remark 6.1. An $\mathrm{SL}_{2}$-equivariant morphism $\varphi: V \rightarrow W$ between two $\mathrm{SL}_{2}$-modules is called a covariant. Every covariant is a sum of homogeneous covariants:

$$
\operatorname{Cov}(V, W)=\bigoplus_{j \in \mathbb{N}} \operatorname{Cov}(V, W)_{j}
$$

$\operatorname{Cov}(V, W)$ is a finitely generated $\mathcal{O}(V)^{\mathrm{SL}_{2}}$-module where the module structure is given by $f \varphi(v):=f(v)$.

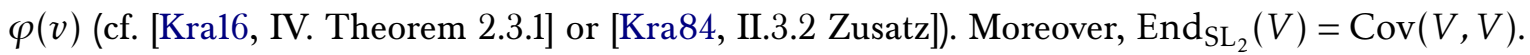

Proposition 6.2. Set $\mathcal{E}_{d}:=\operatorname{End}_{\mathrm{SL}_{2}}\left(V_{d}\right)$, and denote by $J_{d}:=\mathcal{O}\left(V_{d}\right)^{\mathrm{SL}_{2}}$ the algebra of invariants.

(1) $\mathcal{E}_{1}=\mathbb{k i d}_{V_{1}}$, hence $d_{\mathcal{E}_{1}}\left(V_{1}\right)=1$. Moreover, $V_{1}^{\prime}=V_{1} \backslash\{0\}, V_{1} / / \mathcal{E}_{1}=\mathbb{P}\left(V_{1}\right)$ and $\mathcal{F}_{\mathrm{SL}_{2}}\left(V_{1}\right) \simeq \mathbb{k}\left(\mathrm{SL}_{2} / B\right)$.

(2) $\mathcal{E}_{2}=J_{2} \mathrm{id}_{V_{2}}$, hence $d_{\mathcal{E}_{2}}\left(V_{2}\right)=1$. Moreover, $V_{2}^{\prime}=V_{2} \backslash\{0\}, V_{2} / / \mathcal{E}_{2}=\mathbb{P}\left(V_{2}\right)$ and $\mathcal{F}_{\mathrm{SL}_{2}}\left(V_{2}\right) \simeq \mathbb{k}\left(\mathrm{SL}_{2} / N\right)$. 
(3) $\mathcal{E}_{3}=J_{3} \mathrm{id}_{V_{3}} \oplus J_{3} d D$ where $D$ is the discriminant and $d D: V_{3} \rightarrow V_{3}^{*}$ its differential. Hence $d_{\mathcal{E}_{3}}\left(V_{3}\right)=2$. Moreover, $V_{3}^{\prime}=V_{3} \backslash \overline{\mathrm{SL}_{2} \cdot x^{3}}$ and $\mathcal{F}_{\mathrm{SL}_{2}}\left(V_{3}\right) \simeq \mathbb{k}\left(\mathrm{SL}_{2} / N\right)$.

(4) $\mathcal{E}_{4}=J_{4} \mathrm{id}_{V_{4}} \oplus J_{4} H$ where $H$ is the Hessian, hence $d_{\mathcal{E}_{4}}\left(V_{4}\right)=2$. Moreover, $V_{4}^{\prime}=V \backslash \overline{\mathrm{SL}_{2} \cdot \mathrm{k} x^{2} y^{2}}$ and $\mathcal{F}_{\mathrm{SL}_{2}}\left(V_{4}\right) \simeq \mathbb{k}\left(\mathrm{SL}_{2} / O\right)$ where $O$ is the binary octahedral group.

(5) For $d \geq 5$, we have $\mathcal{E}_{d}(f)=V_{d}$ for a generic $f \in V_{d}$, hence $d_{\mathcal{E}_{d}}\left(V_{d}\right)=\operatorname{dim} V_{d}$ and $\mathcal{F}_{\mathrm{SL}_{2}}\left(V_{d}\right)=\mathbb{k}$.

Proof. For $d \leq 4$ the $J_{d}$-module $\mathcal{E}_{d}=\operatorname{Cov}\left(V_{d}, V_{d}\right)$ it is a free module, and the generators can be found in the classical literature, e.g. in [Sch68, II. §8]. For $d=1$ there is a dense orbit isomorphic to $\mathrm{SL}_{2} / U$ whose complement is $\{0\}$. In particular, $V_{1}^{\prime}=V_{1} \backslash\{0\}$. Since $\mathcal{E}_{1}=\mathbb{k i d}_{V_{1}}$ we get $\mathcal{E}_{1}(f)=\mathbb{k} f$ and so $V_{1} / / \mathcal{E}_{1}=\mathbb{P}\left(V_{1}\right)$. The remaining claims of (1) follow from Proposition 5.17.

For $d>1$ the generic fibers of the quotient maps $\pi: V_{d} \rightarrow V_{d} / / \mathrm{SL}_{2}$ are orbits isomorphic to $\mathrm{SL}_{2} / H_{d}$ where $H_{2}=T, H_{3}=\mu_{3}, H_{4}=\tilde{D}_{4}$, the binary dihedral group of order 8 . In the first two cases, the normalizer is equal to $N$, and we get $V_{2}^{\prime}=V_{2} \backslash\{0\}$ and $V_{3}^{\prime}=V_{3} \backslash \overline{\mathrm{SL}_{2} x^{3}}$. The remaining claims of (2) and (3) follow from Proposition 5.17 where in (2) we use again the fact that $\mathcal{E}_{2}(f)=\mathbb{k} f$ for a general $f$ to get $V_{2} / / \mathcal{E}_{2}=\mathbb{P}\left(V_{2}\right)$.

For $d=4$ the normalizer of $H_{4}=\tilde{D}_{4}$ is the binary octahedral group $O$ of order 48 and we have $V_{4}^{\prime}=V \backslash \overline{\mathrm{SL}_{2} \cdot \mathrm{k} x^{2} y^{2}}$. Hence $\mathcal{F}_{\mathrm{SL}_{2}}\left(V_{4}\right) \simeq \mathbb{k}\left(\mathrm{SL}_{2} / O\right)$ by Proposition 5.17, proving (4).

For $d>4$ the stabilizer $H_{d}$ is trivial for odd $d$ and equal to the kernel $\{ \pm E\}$ of the action for even $d$. Hence, by Proposition 4.10, $\mathcal{E}(f)=V_{d}$ for a generic $f$, and the claims follow.

\subsection{The nullcone $\mathcal{N}(V)$}

A very interesting object in this setting is the nullcone $\mathcal{N}(V) \subseteq V$ of a representation $V$ of $\mathrm{SL}_{2}$ which is defined in the following way. Denote by $q: V \rightarrow V / / \mathrm{SL}_{2}$ the quotient morphism, i.e., $V / / \mathrm{SL}_{2}=\operatorname{Spec} \mathcal{O}(V)^{\mathrm{SL}_{2}}$ and $q$ is induced by the inclusion $\mathcal{O}(V)^{\mathrm{SL}_{2}} \subseteq \mathcal{O}(V)$. Then $\mathcal{N}(V):=q^{-1}(q(0))$, or equivalently, $\mathcal{N}(V)$ is the zero set of all homogeneous invariants of positive degree. In case $V=V_{d}$ the elements from $\mathcal{N}\left(V_{d}\right)$ are classically called nullforms. One has the following description. Denote by $T \subseteq \mathrm{SL}_{2}$ the diagonal torus, and define the weight spaces

$$
V[i]:=\left\{f \in V \mid\left[\begin{array}{cc}
t & 0 \\
0 & t^{-1}
\end{array}\right] f=t^{i} f \text { for all } t \in \mathbb{k}^{*}\right\} \text { for } i \in \mathbb{N} .
$$

Since the representation of $T$ is completely reducible we have $V=\bigoplus_{j} V[j]$. For $V=V_{d}$ we get the decomposition $V_{d}=\bigoplus_{i=0}^{d} V_{d}[d-2 i]$, and the weight spaces are one-dimensional. Note that $\left[\begin{array}{cc}t & 0 \\ 0 & t^{-1}\end{array}\right] x=t^{-1} x$, and $\left[\begin{array}{cc}t & 0 \\ 0 & t^{-1}\end{array}\right] y=t y$, and so

$$
V_{d}[d-2 i]=\mathbb{k} x^{i} y^{d-i}
$$

Lemma 6.3. The following statements for a form $f \in V_{d}$ are equivalent.

(i) $f$ is a nullform, i.e. $f \in \mathcal{N}\left(V_{d}\right)$.

(ii) There is a one-parameter subgroup $\lambda: \mathbb{k}^{*} \rightarrow \mathrm{SL}_{2}$ such that $\lim _{t \rightarrow 0} \lambda(t) f=0$.

(iii) $f$ is in the $\mathrm{SL}_{2}$-orbit of an element from $V_{d}^{+}:=\bigoplus_{i>0} V_{d}[i] \subseteq V_{d}$.

(iv) $f$ contains a linear factor with multiplicity $>\frac{d}{2}$.

Proof. (a) The equivalence of (i) and (ii) is a consequence of the famous Hilbert-Mumford-Criterion and holds for any representation of a reductive group.

(b) (ii) and (iii) are equivalent, because every one-parameter subgroup of $\mathrm{SL}_{2}$ is conjugate to a oneparameter subgroup of $T$. This holds for any representation of $\mathrm{SL}_{2}$.

(c) The equivalence of (iii) and (iv) is clear, because $V_{d}^{+}$are the forms which contain $y$ with multiplicity at least $\frac{d}{2}$. 
Let $V$ be a representation of $\mathrm{SL}_{2}$. If $\varphi \in \operatorname{End}_{\mathrm{SL}_{2}}(V)$ is homogeneous of degree $k$, then $\varphi(V[j]) \subseteq V[k j]$. It follows that $\varphi\left(\bigoplus_{j \geq j_{0}} V[j]\right) \subseteq \bigoplus_{j \geq k j_{0}} V[j]$. In particular, the subspaces $\bigoplus_{j \geq j_{0}} V[j]$ are $\mathrm{SL}_{2}$-symmetric for any $j_{0} \geq 0$, because any endomorphism is a sum of homogeneous endomorphisms (Remark 6.1). Since every element $f \in \mathcal{N}(V)$ is $\mathrm{SL}_{2}$-equivalent to an element from $V^{+}:=\bigoplus_{j>0} V[j]$ it suffices to study the $\mathrm{SL}_{2}$-symmetric subspaces of $V^{+}$.

\subsection{Special covariants}

For the study of the $\mathrm{SL}_{2}$-symmetric subspaces of the nullforms $\mathcal{N}\left(V_{d}\right)$ we need the existence (and the non-vanishing) of certain covariants which we are going to construct now.

Let $\varphi: V_{d} \rightarrow \operatorname{End}\left(V_{d}\right)$ and $\psi: V_{d} \rightarrow V_{d}$ be homogeneous covariants. Then we define covariants denoted $\Phi_{s}=\Phi_{s}(\varphi, \psi) \in \operatorname{End}_{\mathrm{SL}_{2}}\left(V_{d}\right)$ by

$$
\Phi_{s}(\varphi, \psi) f:=\varphi(f)^{s} \psi(f)=(\varphi(f) \circ \varphi(f) \circ \cdots \circ \varphi(f))(\psi(f))
$$

This is a homogeneous covariant of degree $\operatorname{deg} \Phi_{s}=s \operatorname{deg} \varphi+\operatorname{deg} \psi$.

Let $\mathfrak{s l}_{2}:=\mathrm{LieSL}_{2}$ be the Lie algebra of $\mathrm{SL}_{2}$ which acts on a representation $V$ of $\mathrm{SL}_{2}$ by the adjoint representation ad: $\mathfrak{s l}_{2} \rightarrow \operatorname{End}(V)$. As an $\mathrm{SL}_{2}$-module we have $\mathfrak{s l}_{2} \stackrel{\sim}{\rightarrow} V_{2}$, and $\mathfrak{s l}_{2}[2]=\mathbb{k}\left[\begin{array}{ll}0 & 1 \\ 0 & 0\end{array}\right]$.

Lemma 6.4. Let $V_{d}$ denote the binary forms of degree $d$, considered as a representation of $\mathrm{SL}_{2}$.

(1) If $d$ is odd, then there is a quadratic covariant $\varphi_{0}: V_{d} \rightarrow \mathfrak{s l}_{2}$ such that $\varphi_{0}\left(V_{d}[1]\right)=\mathfrak{s l _ { 2 }}[2]=\mathbb{k}\left[\begin{array}{ll}0 & 1 \\ 0 & 0\end{array}\right]$.

(2) If $d$ is even, then there is a quadratic covariant $\varphi_{0}: V_{d} \rightarrow \mathfrak{s} l_{2} \otimes \mathfrak{s} l_{2}$ such that $\varphi_{0}\left(V_{d}[2]\right)=\mathfrak{s l}_{2}[2] \otimes \mathfrak{s} l_{2}[2]$.

(3) If $d \equiv 0 \bmod 4$, then there is a quadratic covariant $\psi: V_{d} \rightarrow V_{d}$ such that $\psi\left(V_{d}[2]\right)=V_{d}[4]$.

(4) If $d \equiv 2 \bmod 4$ and $d \geq 10$, then there is a homogeneous covariant $\psi: V_{d} \rightarrow V_{d}$ of degree 4 such that $\psi\left(V_{d}[2]\right)=V_{d}[8]$, and there is no quadratic covariant.

For the proof let us recall the Clebsch-Gordan-decomposition of the tensor product $V_{d} \otimes V_{e}$ as an $\mathrm{SL}_{2}$-module where we assume that $d \geq e$ :

$$
V_{d} \otimes V_{e} \simeq \bigoplus_{r=0}^{e} V_{d+e-2 r}
$$

The projection $V_{d} \otimes V_{e} \rightarrow V_{d+e-2 r}$ is classically called the $r$ th transvection. It is given by the following formula:

$$
f \otimes h \mapsto(f, h)_{r}:=\sum_{i=0}^{r}(-1)^{i}\left(\begin{array}{l}
r \\
i
\end{array}\right) \frac{\partial^{r} f}{\partial x^{r-i} \partial y^{i}} \frac{\partial^{r} h}{\partial x^{i} \partial y^{r-i}} .
$$

The second symmetric power $S^{2}\left(V_{d}\right)$ has the decomposition

$$
S^{2}\left(V_{d}\right) \stackrel{\sim}{\rightarrow} V_{2 d} \oplus V_{2 d-4} \oplus V_{2 d-8} \oplus \cdots .
$$

Therefore, the quadratic covariants $\tau_{r}: V_{d} \rightarrow V_{2 d-2 r}, f \mapsto(f, f)_{r}$, are non-zero only for even $r$, and they are given by

$$
\tau_{r}(f)=(f, f)_{r}=\sum_{i=0}^{r}(-1)^{i}\left(\begin{array}{l}
r \\
i
\end{array}\right) \frac{\partial^{r} f}{\partial x^{r-i} \partial y^{i}} \frac{\partial^{r} f}{\partial x^{i} \partial y^{r-i}} \in V_{2 d-2 r} .
$$

For the non-vanishing of the covariant $\tau_{r}$ on the nullforms the following lemma is crucial.

Lemma 6.5. For $d=2 m$ and an even $r=2 s<d$, the transvection

$$
\tau_{2 s}\left(x^{m-1} y^{m+1}\right)=\left(x^{m-1} y^{m+1}, x^{m-1} y^{m+1}\right)_{2 s} \in V_{2 d-4 s}
$$

is equal to $c_{m, 2 s} \cdot x^{d-2 s-2} y^{d-2 s+2}$ where

$$
c_{m, 2 s}=(-1)^{s}(2 s) !(s !)^{2}\left(\begin{array}{c}
m-1 \\
s
\end{array}\right)\left(\begin{array}{c}
m+1 \\
s
\end{array}\right)\left(\begin{array}{c}
2 m-s \\
s
\end{array}\right) \neq 0 .
$$


For the proof we will need some properties of the hypergeometric function ${ }_{3} F_{2}\left(a_{1}, a_{2}, a_{3} ; b_{1}, b_{2} ; z\right)$ which we discuss in the following Section 6.4. The proof of the lemma is then given in Section 6.5.

Proof of Lemma 6.4. As above, $\tau_{r}: V_{d} \rightarrow V_{2 d-2 r}$ denotes the quadratic covariant $f \mapsto(f, f)_{r}$ which is nonzero only for even $r$.

(a) If $d=2 m+1$, then $\tau_{2 m}: V_{d} \rightarrow V_{2} \simeq \mathfrak{s l} 2$, and $\tau_{2 m}\left(x^{m} y^{m+1}\right)$ is a non-zero multiple of $y^{2}$. In fact, for $r=2 m$, the sum (T2) has a single term, namely for $i=m$. This proves (1).

(b) Now assume that $d$ is even, $d=2 m$. Then $\tau_{2 m-2}: V_{d} \rightarrow V_{4}$ has the property that $\tau_{2 m-2}\left(x^{m-1} y^{m+1}\right)$ is a non-zero multiple of $y^{4} \in V_{4}$ [4]. In fact, the sum (T2) has a single term, namely for $i=m-1$. Since $\mathfrak{s l}_{2} \otimes \mathfrak{s l}_{2} \simeq V_{0} \oplus V_{2} \oplus V_{4}$ and $\left(\mathfrak{s l}_{2} \otimes \mathfrak{s l}_{2}\right)[4]=\mathfrak{s l}_{2}[2] \otimes \mathfrak{s l}_{2}[2] \simeq V_{4}[4]$, we thus get $\varphi_{0}: V_{d} \rightarrow \mathfrak{s l}_{2} \otimes \mathfrak{s l}_{2}$ a covariant with the property claimed in (2).

(c) If $d=2 m$ and $m$ even, then, by Lemma $6.5, \tau_{m}: V_{d} \rightarrow V_{d}$ is a quadratic covariant such that $\tau_{m}\left(V_{d}[2]\right)=V_{d}[4]$, proving (3).

(d) Finally, if $d=2 m$ and $m=2 k+1$ is odd, then there is no quadratic covariant, because $V_{d}$ does not appear in the decomposition of $S^{2}\left(V_{d}\right)$. But, for $m \geq 5$, there is a homogeneous covariant $\psi$ of degree 4 with the required property. For even $k$ we take

$$
\psi: V_{d} \rightarrow V_{d}, f \mapsto\left((f, f)_{3 k},(f, f)_{3 k+2}\right)_{1},
$$

and for odd $k$

$$
\psi: V_{d} \rightarrow V_{d}, f \mapsto\left((f, f)_{3 k-1},(f, f)_{3 k+3}\right)_{1} .
$$

By Lemma $6.5,\left(x^{m-1} y^{m+1}, x^{m-1} y^{m+1}\right)_{r}$ is a nonzero multiple of $x^{2 m-r-2} y^{2 m-r+2}$ for every even $r<2 m$. It remains to see that the transvections $\left(x^{k} y^{k+4}, x^{k-2} y^{k+2}\right)_{1}$ for even $k$ and $\left(x^{k+1} y^{k+5}, x^{k-3} y^{k+1}\right)_{1}$ for odd $k$ are nonzero. This follows from the transvection formula (T1) above which gives

$$
\begin{gathered}
\left(x^{k} y^{k+4}, x^{k-2} y^{k+2}\right)_{1}=8 \cdot x^{2 k-3} y^{2 k+5}=8 \cdot x^{m-4} y^{m+4}, \\
\left(x^{k+1} y^{k+5}, x^{k-3} y^{k+1}\right)_{1}=16 \cdot x^{2 k-3} y^{2 k+5}=16 \cdot x^{m-4} y^{m+4} .
\end{gathered}
$$

This proves (4).

\subsection{The hypergeometric function ${ }_{3} F_{2}$}

The Pochhammer function $(z)_{n}:=z(z+1) \cdots(z+n-1)$ is defined for $z \in \mathbb{C}$ and any integer $n \geq 0$ where we set $(z)_{0}=1$. Note that $(z)_{n}=0$ if $z$ is a negative integer $>-n$. The hypergeometric function ${ }_{3} F_{2}\left(a_{1}, a_{2}, a_{3} ; b_{1}, b_{2} ; z\right)$ is defined by the following convergent series

$$
{ }_{3} F_{2}\left(a_{1}, a_{2}, a_{3} ; b_{1}, b_{2} ; z\right):=\sum_{k=0}^{\infty} \frac{\left(a_{1}\right)_{k}\left(a_{2}\right)_{k}\left(a_{3}\right)_{k}}{\left(b_{1}\right)_{k}\left(b_{2}\right)_{k}} \frac{z^{k}}{k !}
$$

where $a_{1}, a_{2}, a_{3}, b_{1}, b_{2} \in \mathbb{C}$ and $b_{1}, b_{2} \notin\{0,-1,-2,-3, \ldots\}$, see [Sla66]. The ${ }_{3} F_{2}$-series can be evaluated by means of Dixon's summation formula (see [Sla66, formula 2.3.3.6 on page 52]):

$$
{ }_{3} F_{2}(a, b,-n ; 1+a-b, 1+a+n ; 1)=\frac{(1+a)_{n}\left(1+\frac{a}{2}-b\right)_{n}}{\left(1+\frac{a}{2}\right)_{n}(1+a-b)_{n}},
$$

where $n$ is a nonnegative integer. As mentioned above, the series is well-defined if neither $1+a-b$ nor $1+a+n$ belong to $\{0,-1,-2,-3, \ldots\}$. The right-hand-side is a rational function in $a, b$, namely a quotient of products of linear terms, and there is some cancellation in the quotient $\frac{(1+a)_{n}}{\left(1+\frac{a}{2}\right)_{n}}$ if $n>1$, e.g. $\frac{(1+a)_{2}}{\left(1+\frac{a}{2}\right)_{2}}=\frac{(1+a)(2+a)}{\left(1+\frac{a}{2}\right)\left(2+\frac{a}{2}\right)}=\frac{2(1+a)}{2+\frac{a}{2}}$. 
More precisely, setting $a=2 z$, we find

$$
\begin{aligned}
\frac{(1+2 z)_{n}}{(1+z)_{n}} & =\frac{(2 z+1)(2 z+2) \cdots(2 z+n)}{(z+1)(z+2) \cdots(z+n)}= \\
& = \begin{cases}\frac{(2 z+1)(2 z+3) \cdots(2 z+2 \ell+1) 2^{\ell}}{(z+\ell+1)(z+\ell+2) \cdots(z+2 \ell+1)} & \text { if } n=2 \ell+1 \text { is odd, } \\
\frac{(2 z+1)(2 z+3) \cdots(2 z+2 \ell-1) 2^{\ell}}{(z+\ell+1)(z+\ell+2) \cdots(z+2 \ell)} & \text { if } n=2 \ell \text { is even. }\end{cases}
\end{aligned}
$$

This shows that the poles of the right hand side of $(*)$ are the even integers $a$ such that $-\ell-1 \geq \frac{a}{2} \geq-n$. But this implies that $1+a+n$ is a negative integer, and these values are excluded in the definition of ${ }_{3} F_{2}$.

\subsection{Proof of Lemma 6.5}

The following proof was communicated to us by Christian Krattenthaler. From formula (T2) we get

$$
c_{m, r}=\sum_{i=0}^{r}(-1)^{i}\left(\begin{array}{l}
r \\
i
\end{array}\right)(m-r+i)_{r-i}(m-r+i+2)_{r-i}(m-i+2)_{i}(m-i)_{i},
$$

It follows that for a fixed integer $r \geq 0$ the coefficient $c_{m, r}$ is a polynomial in $m$, and the same holds for the claimed expression of $c_{m, 2 s}$ given in Lemma 6.5 above. Therefore, for a given $r=2 s$, it suffices to prove the equality for infinitely many $m$. We will do this for all integers $m \geq r+1$ what we assume from now on.

Using the following obvious identities for the Pochhammer function $(z)_{n}$

$$
\begin{gathered}
\frac{(m-r+i)_{r-i}}{(m-r)_{r}}=\frac{1}{(m-r)_{i}}, \quad \frac{(m-r+i+2)_{r-i}}{(m-r+2)_{r}}=\frac{1}{(m-r+2)_{i}}, \\
(m-i)_{i}=(-1)^{i}(-m+1)_{i} \quad(m-i+2)_{i}=(-1)^{i}(-m-1)_{i}, \\
\left(\begin{array}{l}
r \\
i
\end{array}\right)=\frac{1}{i !}(r-i+1)_{i}=(-1)^{i} \frac{1}{i !}(-r)_{i},
\end{gathered}
$$

we find for the summands in $(* *)$

$$
(-1)^{i}\left(\begin{array}{l}
r \\
i
\end{array}\right)(m-r+i)_{r-i}(m-r+i+2)_{r-i}(m-i+2)_{i}(m-i)_{i}=(m-r)_{r}(m-r+2)_{r} \frac{1}{i !} \frac{(-r)_{i}(-m-1)_{i}(-m+1)_{i}}{(m-r+2)_{i}(m-r)_{i}},
$$

hence

$$
c_{m, r}=(m-r)_{r}(m-r+2)_{r}{ }_{3} F_{2}(-r,-m-1,-m+1 ; m-r+2, m-r ; 1) .
$$

The ${ }_{3} F_{2}$-series can be evaluated by means of Dixon's summation formula $(*)$ above where $a=-r, b=-m-1$, $n=m-1$ :

$$
{ }_{3} F_{2}(-r,-m-1,-m+1 ; m-r+2, m-r ; 1)=\frac{(1-r)_{m-1}(m+2-r / 2)_{m-1}}{(1-r / 2)_{m-1}(m+2-r)_{m-1}} .
$$

As we have seen above this equality holds for a positive integer $m \geq 1$ and any $r \in \mathbb{C}$ as long as $m-r$ is not a negative integer. Setting $r=2 s$ we get (see the calculation in Section 6.4 above):

$$
\begin{aligned}
\frac{(1-2 s)_{m-1}}{(1-s)_{m-1}} & =\frac{(2 s-1)(2 s-2) \cdots(2 s-(m-1))}{(s-1)(s-2) \cdots(s-(m-1))}= \\
& = \begin{cases}\frac{(2 s-1)(2 s-3) \cdots(2 s-2 \ell+1) 2^{\ell-1}}{(s-\ell)(s-\ell-1) \cdots(s-2 \ell+1)} & \text { if } m=2 \ell \text { is even, } \\
\frac{(2 s-1)(2 s-3) \cdots(2 s-2 \ell+3) 2^{\ell-1}}{(s-\ell)(s-\ell-1) \cdots(s-2 \ell+2)} & \text { if } m=2 \ell-1 \text { is odd. }\end{cases}
\end{aligned}
$$


Hence, this fraction is well-defined in the given range $r=2 s \leq m-1$, since this means that $s \leq \ell-1$ in both cases, and so all factors in the denominator are strictly negative integers. In both cases the denominator can be written as $(-1)^{\ell} \frac{(m-s-1) !}{(\ell-s-1) !}$. For the numerator, we find in case $m=2 \ell$ :

$$
\begin{aligned}
& 2^{\ell-1}(2 s-1)(2 s-3) \cdots 3 \cdot 1 \cdot(-1) \cdot(-3) \cdots(-(2 \ell-2 s-1))= \\
& \quad=2^{\ell-1} \frac{(2 s) !}{2^{s} \cdot s !} \cdot(-1)^{\ell-s} \frac{(2 \ell-2 s) !}{2^{\ell-s}(l-s) !}=\frac{(-1)^{\ell-s}(2 s) !(2 \ell-2 s) !}{2 s !(\ell-s) !},
\end{aligned}
$$

and for $m=2 \ell-1$ :

$$
\begin{aligned}
& 2^{\ell-1}(2 s-1)(2 s-3) \cdots 3 \cdot 1 \cdot(-1) \cdot(-3) \cdots(-(2 \ell-2 s-3))= \\
& \quad=2^{\ell-1} \frac{(2 s) !}{2^{s} \cdot s !} \cdot(-1)^{\ell-s} \frac{(2 \ell-2 s-2) !}{2^{\ell-s-1}(l-s-1) !}=\frac{(-1)^{\ell-s}(2 s) !(2 \ell-2 s-2) !}{s !(\ell-s-1) !} .
\end{aligned}
$$

This gives for the right hand side of $(* * *)$ for an even $m=2 \ell$

$$
(-1)^{s} \frac{(2 s) !(2 \ell-2 s) !}{2 s !(\ell-s) !} \cdot \frac{(\ell-s-1) !}{(m-s-1) !}=(-1)^{s} \frac{(2 s) !(m-2 s-1) !}{s !(m-s-1) !},
$$

and the same for an odd $m=2 \ell-1$

$$
(-1)^{s} \frac{(2 s) !(2 \ell-2 s-2) !}{s !(\ell-s-1) !} \cdot \frac{(\ell-s-1) !}{(m-s-1) !}=(-1)^{s} \frac{(2 s) !(m-2 s-1) !}{s !(m-s-1) !} .
$$

The remaining factors are

$$
\begin{aligned}
(m-r+2)_{r}(m-r)_{r} \frac{(m+2-r / 2)_{m-1}}{(m+2-r)_{m-1}} & =\frac{(m+1) !}{(m-2 s+1) !} \frac{(m-1) !}{(m-2 s-1) !} \frac{(2 m-s) !}{(m-s+1) !} \frac{(m-2 s+1) !}{(2 m-2 s) !} \\
& =\frac{(m+1) !(m-1) !(2 m-s) !}{(m-2 s-1) !(m-s+1) !(2 m-2 s) !} .
\end{aligned}
$$

Hence

$$
\begin{aligned}
c_{m, 2 s} & =(-1)^{s} \frac{(2 s) !(m-2 s-1) !}{s !(m-s-1) !} \cdot \frac{(m+1) !(m-1) !(2 m-s) !}{(m-2 s-1) !(m-s-1) !(2 m-2 s) !} \\
& =(-1)^{s} \frac{(2 s) !(m+1) !(m-1) !(2 m-s) !}{s !(m-s-1) !(m-s+1) !(2 m-2 s) !} \\
& =(-1)^{s}(2 s) !(s !)^{2}\left(\begin{array}{c}
m-1 \\
s
\end{array}\right)\left(\begin{array}{c}
m+1 \\
s
\end{array}\right)\left(\begin{array}{c}
2 m-s \\
s
\end{array}\right),
\end{aligned}
$$

as claimed.

\section{6. $\mathrm{Vec}_{\mathrm{SL}_{2}}$-symmetric subspaces of the nullforms}

We will now determine the minimal $\mathrm{Vec}_{\mathrm{SL}_{2}}$-symmetric subspaces of the nullforms $\mathcal{N}\left(V_{d}\right)$ and calculate the first integrals.

Proposition 6.6. Let $d=2 m+1$ be odd, $d \geq 3$.

(1) $d\left(\mathcal{N}\left(V_{d}\right)\right)=m$.

(2) $V_{d}^{+}$is a minimal $\mathrm{SL}_{2}$-symmetric subspace of $\mathcal{N}\left(V_{d}\right)$ of dimension $m$.

(3) If $M \subseteq \mathcal{N}\left(V_{d}\right)$ is a minimal $\mathrm{SL}_{2}$-symmetric subspace of dimension $m$, then $M=g V_{d}^{+}$for some $g \in \mathrm{SL}_{2}$.

(4) $\mathcal{N}\left(V_{d}\right) / / \operatorname{End}_{\mathrm{SL}_{2}}\left(V_{d}\right) \simeq \mathrm{SL}_{2} / B \simeq \mathbb{P}^{1}$.

(5) $\mathcal{F}_{\mathrm{SL}_{2}}\left(\mathcal{N}\left(V_{d}\right)\right) \simeq \mathbb{k}\left(\mathrm{SL}_{2} / B\right)$, in particular $\mathcal{F}_{\mathrm{SL}_{2}}\left(\mathcal{N}\left(V_{d}\right)\right)^{\mathrm{SL}_{2}}=\mathbb{k}$ 
Proof. (a) Consider the covariants $\Phi_{s}(\varphi$, id $): V_{d} \rightarrow V_{d}$ defined above where $\varphi$ is the composition

$$
\varphi: V_{d} \stackrel{\varphi_{0}}{\longrightarrow} \mathfrak{s l}_{2} \stackrel{\text { ad }}{\longrightarrow} \operatorname{End}\left(V_{d}\right)
$$

and $\varphi_{0}: V_{d} \rightarrow \mathfrak{s l}_{2}$ is from Lemma 6.4(1). By construction, we get

$$
\Phi_{s}\left(V_{d}[1]\right)=\operatorname{ad}\left[\begin{array}{ll}
0 & 1 \\
0 & 0
\end{array}\right]^{s} V_{d}[1]=V_{d}[2 s+1] .
$$

This shows that $\operatorname{End}_{\mathrm{SL}_{2}}\left(V_{d}\right)\left(V_{d}[1]\right)=V_{d}^{+}$, hence (1) and (2).

(b) Let $M=M(f)$ be of dimension $m$. There is a $g \in \mathrm{SL}_{2}$ such that $g f \in V_{d}^{+}$, hence $g M(f)=M(g f)$ is contained in $V_{d}^{+}$. Since $\operatorname{dim} M(f)=m$ we get $g M(f)=V_{d}^{+}$. This gives (3) and shows that $\mathrm{SL}_{2}$ acts transitively on the subspaces $M(f) \subseteq \mathcal{N}\left(V_{d}\right)$ of dimension $m$, thus on the image of $\pi: \mathcal{N}\left(V_{d}\right) \rightarrow \mathrm{Gr}_{m}\left(V_{d}\right)$. Since the normalizer of $V_{d}^{+}$is $B$, we finally get (4) and (5).

Proposition 6.7. Let $d=2 m$ and $m$ even.

(1) $d\left(\mathcal{N}\left(V_{d}\right)\right)=m$.

(2) $V_{d}^{+}$is a minimal $\mathrm{SL}_{2}$-symmetric subspace of $\mathcal{N}\left(V_{d}\right)$ of dimension $m$.

(3) If $M \subseteq \mathcal{N}\left(V_{d}\right)$ is a minimal $\mathrm{SL}_{2}$-symmetric subspace of dimension $m$, then $M=g V_{d}^{+}$for some $g \in \mathrm{SL}_{2}$.

(4) $\mathcal{N}\left(V_{d}\right) / / \operatorname{End}_{\mathrm{SL}_{2}}\left(V_{d}\right) \simeq \mathrm{SL}_{2} / B \simeq \mathbb{P}^{1}$.

(5) $\mathcal{F}_{\mathrm{SL}_{2}}\left(\mathcal{N}\left(V_{d}\right)\right) \simeq \mathbb{k}\left(\mathrm{SL}_{2} / B\right)$, in particular $\mathcal{F}_{\mathrm{SL}_{2}}\left(\mathcal{N}\left(V_{d}\right)\right)^{\mathrm{SL}_{2}}=\mathbb{k}$.

Proof. Define the following covariant

$$
\varphi: V_{d} \stackrel{\varphi_{0}}{\longrightarrow} \mathfrak{s l}_{2} \otimes \mathfrak{s l}_{2} \stackrel{\alpha}{\longrightarrow} \operatorname{End}\left(V_{d}\right)
$$

where $\varphi_{0}$ is from Lemma 6.4(2), and $\alpha$ is the linear $\mathrm{SL}_{2}$-equivariant map $A \otimes B \mapsto \operatorname{ad} A \circ \operatorname{ad} B$. Then the covariants $\Phi_{s}(\varphi, \mathrm{id}): V_{d} \rightarrow V_{d}$ satisfy $\Phi_{s}\left(V_{d}[2]\right)=\left(\operatorname{ad}\left[\begin{array}{ll}0 & 1 \\ 0 & 0\end{array}\right]\right)^{2 s} V_{d}[2]=V_{d}[4 s+2]$, and for the covariants $\Phi_{s}(\varphi, \psi)$ where $\psi$ is from Lemma $6.4(3)$ we get $\Phi_{s}\left(V_{d}[2]\right)=\operatorname{ad}\left[\begin{array}{ll}0 & 1 \\ 0 & 0\end{array}\right]^{2 s} V_{d}[4]=V_{d}[4 s+4]$. As a consequence, we get $\operatorname{End}_{\mathrm{SL}_{2}}\left(V_{d}\right)\left(V_{d}[2]\right)=V_{d}^{+}$, hence (1) and (2). The remaining claims follow as in the proof of Proposition 6.6.

If $d=2 m$ and $m$ odd we define $V_{d}^{++}:=V_{d}[2] \oplus V_{d}[6] \oplus V_{d}[8] \oplus \cdots$.

Proposition 6.8. Let $d=2 m$ and $m$ odd, $m \geq 3$.

(1) $d\left(\mathcal{N}\left(V_{d}\right)\right)=m-1$.

(2) $V_{d}^{++}$is a minimal $\mathrm{SL}_{2}$-symmetric subspace of $\mathcal{N}\left(V_{d}\right)$ of dimension $m-1$.

(3) If $M \subseteq \mathcal{N}\left(V_{d}\right)$ is a minimal $\mathrm{SL}_{2}$-symmetric subspace of dimension $m-1$, then $M=g V_{d}^{++}$for some $g \in \mathrm{SL}_{2}$.

(4) $\mathcal{N}\left(V_{d}\right) / / \operatorname{End}_{\mathrm{SL}_{2}}\left(V_{d}\right) \simeq \mathrm{SL}_{2} / T$.

(5) $\mathcal{F}_{\mathrm{SL}_{2}}\left(\mathcal{N}\left(V_{d}\right)\right) \simeq \mathbb{k}\left(\mathrm{SL}_{2} / T\right)$, in particular $\mathcal{F}_{\mathrm{SL}_{2}}\left(\mathcal{N}\left(V_{d}\right)\right)^{\mathrm{SL}_{2}}=\mathbb{k}$.

Proof. (a) We first remark that there is no quadratic covariant $\varphi: V_{d} \rightarrow V_{d}$, and so $V_{d}^{++}$is stable under $\mathcal{E}:=\operatorname{End}_{\mathrm{SL}_{2}}\left(V_{d}\right)$. Now we use the covariants $\Phi_{s}(\varphi$,id), as in the proof of the previous proposition, to show that $\mathcal{E}\left(V_{d}[2]\right) \supset V_{d}[4 s+2]$. Moreover, the covariants $\Phi_{s}(\varphi, \psi)$ with $\psi$ from Lemma 6.4(4) imply that the inclusion $\mathcal{E}\left(V_{d}[2]\right) \supset V_{d}[4 s+8]$ holds. It follows that $\mathcal{E}\left(V_{d}[2]\right)=V_{d}^{++}$, hence (1) and (2).

(b) Using again that there are no quadratic covariants, we see that

$$
\mathcal{E}\left(V_{d}[4]\right) \subseteq V_{d}[4] \oplus V_{d}[8] \oplus V_{d}[10] \oplus \cdots,
$$

hence $\operatorname{dim} \mathcal{E}\left(V_{d}[4]\right) \leq m-2$. Therefore, $V_{d}^{++}$is the only minimal $\mathrm{SL}_{2}$-symmetric subspace of $V_{d}^{+}$of dimension $m-1$. Now the remaining claims follow as before, using that the normalizer of $V_{d}^{++}$is $T$. 
Example 6.9. The minimal orbit $O_{0} \subseteq V_{d}$ is the orbit of $y^{d}$. Denote by $O_{1}$ the orbit of $x y^{d-1}$. Then $X:=\overline{O_{1}}=O_{1} \cup O_{0} \cup\{0\}$. We claim that $X$ is $\mathrm{SL}_{2}$-symmetric and that $\operatorname{End}_{\mathrm{SL}_{2}}(X)=\mathbb{k} \cdot$ id in case $d \geq 5$. In fact, the image of $x y^{d-1} \in V_{d}^{+}$under a homogeneous $\varphi \in \operatorname{End}_{\mathrm{SL}_{2}}(X)$ is again a weight vector of positive weight, hence a multiple of some $x^{\ell} y^{d-\ell}$ where $\ell<d-\ell$. Since the stabilizer of $x^{\ell} y^{d-\ell}$ in $\mathrm{SL}_{2}$ is cyclic of order $d-2 \ell$ for $\ell<d-\ell$, we see that $\varphi\left(x y^{d-1}\right.$ ) is a multiple of $x y^{d-1}$ if $d>4$. (For $d=4, X$ is the nullcone $\mathcal{N}\left(V_{4}\right)$, and the quadratic covariant $\varphi$ sends $O_{1}$ onto $O_{0}$, see Proposition 6.7.) This implies that $\left.\varphi\right|_{O_{1}}=\lambda \cdot$ id for some $\lambda \in \mathbb{k}$, hence $\left.\varphi\right|_{X}=\lambda \cdot$ id. As a consequence, $X^{\prime}=X \backslash\{0\}$, and $X / / \mathcal{E}=\mathbb{P}(X) \subseteq \mathbb{P}\left(V_{d}\right)$.

Example 6.10. Let $d=2 m$ be even and consider $V_{d}^{+}$as a $B$-module. It is not difficult to see that there is always a $B$-covariant $\varphi$ of degree 2. E.g. for $d=6$ it is given by

$$
\varphi\left(a_{1} \cdot x^{2} y^{4}+a_{2} \cdot x y^{5}+a_{3} \cdot y^{6}\right)=2 a_{1}^{2} \cdot x y^{5}+a_{1} a_{2} \cdot y^{6} .
$$

On the other hand, for $d=2 m \geq 6$ and $m$ odd there is no $\mathrm{SL}_{2}$-covariant of $V_{d}$ of degree 2 (Lemma 6.4(4)). Since $\operatorname{End}_{\mathrm{SL}_{2}}(V)=\operatorname{End}_{B}(V)$ for every $\mathrm{SL}_{2}$-module $V$, we see that for $d \equiv 2 \bmod 4$ and $d \geq 6$ the restriction map $\operatorname{End}_{B}\left(V_{d}\right) \rightarrow \operatorname{End}_{B}\left(V_{d}^{+}\right)$is not surjective.

\section{Appendix: Ind-varieties and ind-semigroups}

An introduction to ind-varieties and ind-groups can be found in Kumar's book [Kum02, Chapter IV].

\section{A.1. Basic definitions}

The following is borrowed from [FK18].

Definition A.1. An ind-variety $\mathcal{V}$ is a set together with an ascending filtration $\mathcal{V}_{0} \subseteq \mathcal{V}_{1} \subseteq \mathcal{V}_{2} \subseteq \cdots \subseteq \mathcal{V}$ such that the following holds:

(1) $\mathcal{V}=\bigcup_{k \in \mathbb{N}} \mathcal{V}_{k}$;

(2) Each $\mathcal{V}_{k}$ has the structure of an algebraic variety;

(3) For all $k \in \mathbb{N}$ the inclusion $\mathcal{V}_{k} \hookrightarrow \mathcal{V}_{k+1}$ is a closed immersion of algebraic varieties.

A morphism between ind-varieties $\mathcal{V}$ and $\mathcal{W}$ is a map $\varphi: \mathcal{V} \rightarrow \mathcal{W}$ such that for any $k$ there is an $m$ such that $\varphi\left(\mathcal{V}_{k}\right) \subseteq \mathcal{W}_{m}$ and that the induced map $\mathcal{V}_{k} \rightarrow \mathcal{W}_{m}$ is a morphism of varieties. Isomorphisms of ind-varieties are defined in the obvious way.

Two filtrations $\mathcal{V}=\bigcup_{k \in \mathbb{N}} \mathcal{V}_{k}$ and $\mathcal{V}=\bigcup_{k \in \mathbb{N}} \mathcal{V}_{k}^{\prime}$ are called equivalent if for any $k$ there is an $m$ such that $\mathcal{V}_{k} \subseteq \mathcal{V}_{m}^{\prime}$ is a closed subvariety as well as $\mathcal{V}_{k}^{\prime} \subseteq \mathcal{V}_{m}$. Equivalently, the identity map

$$
\operatorname{id}: \mathcal{V}=\bigcup_{k \in \mathbb{N}} \mathcal{V}_{k} \rightarrow \mathcal{V}=\bigcup_{k \in \mathbb{N}} \mathcal{V}_{k}^{\prime}
$$

is an isomorphism of ind-varieties.

Definition A.2. The Zariski topology of an ind-variety $\mathcal{V}=\bigcup_{k} \mathcal{V}_{k}$ is defined by declaring a subset $U \subseteq \mathcal{V}$ to be open if the intersections $U \cap \mathcal{V}_{k}$ are Zariski-open in $\mathcal{V}_{k}$ for all $k$. It is obvious that $A \subseteq \mathcal{V}$ is closed if and only if $A \cap \mathcal{V}_{k}$ is Zariski-closed in $\mathcal{V}_{k}$ for all $k$. It follows that a locally closed subset $\mathcal{W} \subseteq \mathcal{V}$ has a natural structure of an ind-variety, given by the filtration $\mathcal{W}_{k}:=\mathcal{W} \cap \mathcal{V}_{k}$ which are locally closed subvarieties of $\mathcal{V}_{k}$. These subsets are called ind-subvarieties.

A morphism $\varphi: \mathcal{V} \rightarrow \mathcal{W}$ is called an immersion if the image $\varphi(\mathcal{V}) \subseteq \mathcal{W}$ is locally closed and $\varphi$ induces an isomorphism $\mathcal{V} \stackrel{\sim}{\rightarrow} \varphi(\mathcal{V})$ of ind-varieties. An immersion $\varphi$ is called a closed (resp. open) immersion if $\varphi(\mathcal{V}) \subseteq \mathcal{W}$ is closed (resp. open).

\section{Definition A.3.}


(1) An ind-variety $\mathcal{V}$ is called affine if it admits a filtration such that all $\mathcal{V}_{k}$ are affine. It follows that any filtration of $\mathcal{V}$ has this property.

(2) The algebra of regular functions on $\mathcal{V}=\bigcup \mathcal{V}_{k}$ is defined as

$$
\mathcal{O}(\mathcal{V}):=\operatorname{Mor}\left(\mathcal{V}, \mathbb{A}^{1}\right)=\lim \mathcal{O}\left(\mathcal{V}_{k}\right)
$$

It will always be regarded as a topological algebra with the obvious topology as an inverse limit of finitely generated algebras. The homomorphism $\varphi^{*}: \mathcal{O}(\mathcal{W}) \rightarrow \mathcal{O}(\mathcal{V})$ induced by any morphism $\varphi: \mathcal{V} \rightarrow \mathcal{W}$ is continuous. Moreover, an affine ind-variety $\mathcal{V}$ is uniquely determined by the topological algebra $\mathcal{O}(\mathcal{V})$.

(3) The Zariski tangent space of an ind-variety $\mathcal{V}=\bigcup_{k} \mathcal{V}_{k}$ is defined in the obvious way:

$$
T_{v} \mathcal{V}:=\underset{\lim }{\longrightarrow} T_{v} \mathcal{V}_{k}
$$

If $\mathcal{V}$ is affine, a tangent vector $A \in T_{\mathcal{V}} \mathcal{V}$ is the same as a continuous derivation $A: \mathcal{O}(\mathcal{V}) \rightarrow \mathbb{k}$ in $v$. It is clear that a morphism $\varphi: \mathcal{V} \rightarrow \mathcal{W}$ between two ind-varieties induces a linear map between tangent spaces $d \varphi_{v}: T_{v} \mathcal{V} \rightarrow T_{\varphi(v)} \mathcal{W}$, the differential of $\varphi$ in $v$.

(4) The product of two ind-varieties $\mathcal{V}=\bigcup_{k} \mathcal{V}_{k}$ and $\mathcal{W}=\bigcup_{j} \mathcal{W}_{j}$ is the ind-variety defined as

$$
\mathcal{V} \times \mathcal{W}:=\bigcup_{k} \mathcal{V}_{k} \times \mathcal{W}_{k}
$$

It has the usual universal properties.

(5) An ind-variety $\mathcal{V}$ is curve-connected if for every pair $v, w \in \mathcal{V}$ there is an irreducible algebraic curve $C$ and a morphism $\gamma: C \rightarrow \mathcal{V}$ such that $v, w \in \gamma(C)$. One can show that this is equivalent to the existence of a filtration $\mathcal{V}=\bigcup_{k} \mathcal{V}_{k}$ such that all $\mathcal{V}_{k}$ are irreducible (see [FK18]).

Since products exist in the category of ind-varieties we can define ind-groups and ind-semigroups.

Definition A.4. An ind-group $\mathcal{G}$ is an ind-variety with a group structure such that multiplication $\mathcal{G} \times \mathcal{G} \rightarrow \mathcal{G}$ and inverse $\mathcal{G} \rightarrow \mathcal{G}$ are morphisms. An ind-semigroup $\mathcal{S}$ is defined in a similar way.

An action of an ind-group $\mathcal{G}$ on a variety $X$ is a homomorphism $\mathcal{G} \rightarrow \operatorname{Aut}(X)$ such that the induced map $\mathcal{G} \times X \rightarrow X$ is a morphism of ind-varieties. If $X$ is an affine variety, it is shown in [FK18] that $\operatorname{End}(X)$ is an affine ind-semigroup and $\operatorname{Aut}(X)$ is an affine ind-group which is locally closed in $\operatorname{End}(X)$. It follows that an action of an ind-group $\mathcal{G}$ on $X$ is the same as a homomorphism of ind-groups $\mathcal{G} \rightarrow \operatorname{Aut}(X)$.

All this carries over to actions of ind-semigroups $\mathcal{S}$.

\section{A.2. Vector fields and Lie algebras}

A vector field $\delta$ on an affine variety $X$ is a collection $\delta=(\delta(x))_{x \in X}$ of tangent vectors $\delta(x) \in T_{x} X$ such that, for all $f \in \mathcal{O}(X)$, we have $\delta f \in \mathcal{O}(X)$ where $(\delta f)(x):=\delta(x) f$. It follows that the vector fields $\operatorname{Vec}(X)$ can be identified with the derivations of $\mathcal{O}(X)$ which we denote by $\operatorname{Der}(\mathcal{O}(X))$.

The same definition can be used for an affine ind-variety $\mathcal{V}$, and one gets an identification of $\operatorname{Vec}(\mathcal{V})$ with the continuous derivations $\operatorname{Der}^{c}\left(\mathcal{O}(\mathcal{V})\right.$ ). For an affine ind-group $\mathcal{G}$ one shows that the tangent space $T_{e} \mathcal{G}$ has a natural structure of a Lie algebra. It will be denoted by Lie $\mathcal{G}$.

If $\mathcal{G}$ acts on the variety $X$ and $x \in X$ we denote by $\mu_{x}: \mathcal{G} \rightarrow X$ the orbit map $g \mapsto g x$.

Proposition A.5. Assume that an affine ind-group $\mathcal{G}$ acts on an affine variety $X$. For $A \in$ Lie $\mathcal{G}$ and $x \in X$ define the tangent vector $\xi_{A}(x) \in T_{x} X$ to be the image of $A$ under $d \mu_{x}:$ Lie $\mathcal{G} \rightarrow T_{X} X$. Then $\xi_{A}$ is a vector field on $X$. The resulting linear map $\Xi: \operatorname{Lie} \mathcal{G} \rightarrow \operatorname{Vec}(X), A \mapsto \xi_{A}$, is a anti-homomorphism of Lie algebras. 
Outline of Proof. The action $\varphi: \mathcal{G} \times X \rightarrow X$ defines a homomorphism $\varphi^{*}: \mathcal{O}(X) \rightarrow \mathcal{O}(\mathcal{G}) \otimes \mathcal{O}(X)$. Now consider the following derivation of $\mathcal{O}(X)$ :

$$
\delta: \mathcal{O}(X) \stackrel{\varphi^{*}}{\longrightarrow} \mathcal{O}(\mathcal{G}) \otimes \mathcal{O}(X) \stackrel{A \otimes \mathrm{id}}{\longrightarrow} \mathcal{O}(X) .
$$

An easy calculation shows that $(\delta f)(x)=A \mu_{x}^{*}(f)=d \mu_{x}(A) f$, hence $\delta=\xi_{A}$.

It is easy to see that this generalizes to the action of an affine ind-semigroup $\mathcal{E}$ on an affine variety $X$, $\mu: \mathcal{E} \rightarrow \operatorname{End}(X)$, and defines a linear map $\Xi: T_{\mathrm{id}} \mathcal{E} \rightarrow \operatorname{Vec}(X)$ whose image $\mathcal{D}_{\mathcal{E}}$ are the corresponding vector fields.

\section{References}

[AVE67] E. M. Andreev, E. B. Vinberg, and A. G. Elashvili, Orbits of highest dimension of semisimple linear Lie groups, Funkcional. Anal. i Prilozen. 1 (1967), no. 4, 3-7.

[Dom08] M. Domokos, Covariants and the no-name lemma, J. Lie Theory 18 (2008), no. 4, 839-849.

[Ell64] E. B. Elliott, An Introduction to the Algebra of Quantics, Oxford University Press, 1913; reprinted by Chelsea Publishing Company, 1964.

[FK18] J.-P. Furter and H. Kraft, On the geometry of the automorphism groups of affine varieties, preprint arXiv:1809.04175 (2018).

[GSW12] F. D. Grosshans, J. Scheurle, and S. Walcher, Invariant sets forced by symmetry, J. Geom. Mech. 4 (2012), no. 3, 271-296.

[Hes79] W. Hesselink, The normality of closures of orbits in a Lie algebra, Comment. Math. Helv. 54 (1979), no. $1,105-110$.

[Kra84] H. Kraft, Geometrische Methoden in der Invariantentheorie, Aspects of Mathematics, D1, Friedr. Vieweg \& Sohn, Braunschweig, 1984.

[Kra16] __ Algebraic Transformation Groups: An Introduction, Mathematisches Institut, Universität Basel, 2016. Available at http://kraftadmin.wixsite.com/hpkraft

[Kum02] S. Kumar, Kac-Moody groups, their flag varieties and representation theory, Progress in Mathematics, vol. 204, Birkhäuser Boston Inc., Boston, MA, 2002.

[LS99] G. I. Lehrer and T. A. Springer, A note concerning fixed points of parabolic subgroups of unitary reflection groups, Indag. Math. (N.S.) 10 (1999), no. 4, 549-553.

[Lun73] D. Luna, Slices étales. In: Sur les groupes algébriques, pp. 81-105, Bull. Soc. Math. France, Paris, Mémoire 33, Soc. Math. France, Paris, 1973.

[Pan02] D. I. Panyushev, On covariants of reductive algebraic groups, Indag. Math. (N.S.) 13 (2002), no. 1, 125-129.

[Pop75] A. M. Popov, Irreducible simple linear Lie groups with finite standard subgroups in general position, Funkcional. Anal. i Priložen. 9 (1975), no. 4, 81-82.

[Pop70] V. L. Popov, Criteria for the stability of the action of a semisimple group on the factorial of a manifold, Izv. Akad. Nauk SSSR Ser. Mat. 34 (1970), 523-531; English Translation in Math. USSR-Izvestija 4 (1970), no. 3, 527-535).

[PV94] V. L. Popov and E. B. Vinberg, Invariant theory. In: Algebraic geometry IV (A. N. Parshin and I. R. Shafarevich, eds.), pp. 123-284, Encyclopaedia of Mathematical Sciences, vol. 55, Springer-Verlag, 1994 . 
[SSW15] M. Santoprete, J. Scheurle, and S. Walcher, Motion in a symmetric potential on the hyperbolic plane, Canad. J. Math. 67 (2015), no. 2, 450-480.

[Sch68] I. Schur, Vorlesungen über Invariantentheorie, Bearbeitet und herausgegeben von Helmut Grunsky. Die Grundlehren der mathematischen Wissenschaften, Band 143, Springer-Verlag, Berlin, 1968.

[Sch13] G. W. Schwarz, Vector fields and Luna strata, J. Pure Appl. Algebra 217 (2013), no. 1, 54-58.

[Sei67] A. Seidenberg, Differential ideals in rings of finitely generated type, Amer. J. Math. 89 (1967), 22-42.

[Sla66] L. J. Slater, Generalized Hypergeometric Functions, Cambridge University Press, Cambridge, 1966.

[Spr89] T. A. Springer, Aktionen reduktiver Gruppen auf Varietäten. In: Algebraische Transformationsgruppen und Invariantentheorie, pp. 3-39, DMV Sem., vol. 13, Birkhäuser, Basel, 1989.

[War71] F. W. Warner, Foundations of differentiable manifolds and Lie groups, Scott, Foresman and Co., Glenview, Ill.-London, 1971. 